\title{
Survey of the Visual Exploration and Analysis of Perfusion Data
}

\author{
Bernhard Preim, Steffen Oeltze, Student Member, IEEE Computer Society, Matej Mlejnek, \\ Eduard Gröller, Member, IEEE Computer Society, Anja Hennemuth, and Sarah Behrens
}

\begin{abstract}
Dynamic contrast-enhanced image data (perfusion data) are used to characterize regional tissue perfusion. Perfusion data consist of a sequence of images, acquired after a contrast agent bolus is applied. Perfusion data are used for diagnostic purposes in oncology, ischemic stroke assessment, or myocardial ischemia. The diagnostic evaluation of perfusion data is challenging, since the data are complex and exhibit various artifacts, e.g., motion artifacts. We provide an overview on existing methods to analyze and visualize CT and MR perfusion data. The integrated visualization of several 2D parameter maps, the 3D visualization of parameter volumes, and exploration techniques are discussed. An essential aspect in the diagnosis of perfusion data is the correlation between perfusion data and derived time-intensity curves as well as with other image data, in particular with high-resolution morphologic image data. We discuss visualization support with respect to the three major application areas: ischemic stroke diagnosis, breast tumor diagnosis, and the diagnosis of coronary heart disease.
\end{abstract}

Index Terms-Medical visualization, multiparameter visualization, volume rendering, perfusion data.

\section{INTRODUCTION}

C OMPARED to static image data, where the morphology of anatomic and pathological structures is represented with high spatial resolution, dynamic image data characterize functional processes, such as metabolism and blood flow, which are often essential to detect diseases at an early stage or to discriminate pathologies with very similar morphology.

Important examples of dynamic medical image data are functional MRI, where activations of brain areas are imaged; dynamic PET and SPECT, where the temporal distribution of a radioactive tracer is measured to assess metabolic processes; and perfusion imaging, where the blood flow is measured. We focus on CT and MR perfusion data, which are acquired to support essential diagnostic tasks, e.g., ischemic stroke diagnosis, the assessment of different types and stages of tumors, and the detection and diagnosis of coronary heart disease (CHD).

With modern CT and MRI devices, the effects of perfusion can be measured in high spatial and temporal resolution. In perfusion imaging, the distribution of contrast agents (CAs) is

- B. Preim and S. Oeltze are with the Department of Simulation and Graphics, Faculty of Computer Science, University of Magdeburg, Universitätsplatz 2, D-39106 Magdeburg, Germany. E-mail: \{preim, stoeltze\}@isg.cs.uni-magdeburg.de.

- M. Mlejnek is with AGFA Healthcare, Dietrichsteingasse 8/14, A-1090 Vienna, Austria.E-mail:mio@cg.tuwien.ac.at.

- E. Gröller is with the Institute of Computer Graphics and Algorithms, Vienna University of Technology, Favoritenstrasse 9-11/E186, A-1040Vienna, Austria.E-mail: groeller@cg.tuwien.ac.at.

- A. Hennemuth is with MeVis Research, Universitätsallee 29, D-28359 Bremen, Germany. E-mail: anja.hennemuth@mevis.de.

- S. Behrens is with MeVis Medical Solutions, Universitätsallee 29, D-28359 Bremen, Germany. E-mail: Sarah.Behrens@mevis.de.

Manuscript received 11 May 2007; revised 21 Dec. 2007; accepted 30 June 2008; published online 8 July 2008.

Recommended for acceptance by T. Moeller.

For information on obtaining reprints of this article, please send e-mail to tvcg@computer.org, and reference IEEECS Log Number TVCG-2007-05-0049. Digital Object Identifier no. 10.1109/TVCG.2008.95. registered to assess blood flow and tissue kinetics. Signal intensities after the administration of a CA are recorded. Whether or not a CA is delivered and subsequently absorbed within a particular region, how long it takes until the maximum amount of $\mathrm{CA}$ is delivered as well as other perfusion parameters are determined for medical diagnosis. These parameters are substitutes for physiological parameters such as tumor perfusion and vessel permeability, which, e.g., characterize the malignancy of a tumor [1].

Visual exploration of perfusion data is particularly challenging. It is primarily based on the derived perfusion parameters, which represent features of time-intensity curves (TICs). These parameters are derived for each voxel of the perfusion data and represent a high-dimensional space, usually of five to eight parameters. The correlation between these parameters as well as the local distribution of single perfusion parameters is essential. Since the time dependency is not represented in the perfusion parameters, often perfusion maps along with TICs have to be analyzed. The comprehensible and simultaneous display of these curves and perfusion maps poses considerable challenges for the layout. The visual exploration is also challenging due to the character and the quality of the data: they exhibit various artifacts and thus the visualization also serves the assessment of the reliability of the original data and also the assessment of preprocessed data, where artifacts are reduced. In contrast to static CT data, no absolute scale for the intensity values exists. Therefore, simple visualization techniques with predefined (absolute) settings are not applicable. As a consequence of these difficulties, image processing and visualization have to be tightly integrated and a variety of visualization techniques is needed to detect and characterize important features.

This paper is organized as follows: In Section 2, we give a brief overview on the medical background in selected application areas. In Section 3, we briefly describe the image data processing, which enhances the expressiveness of simple visualization techniques and is indispensable 


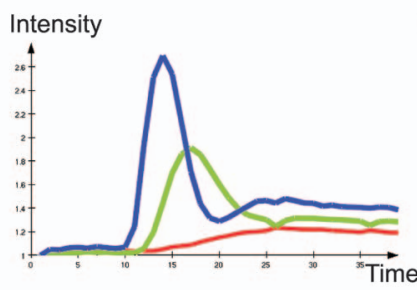

(a)

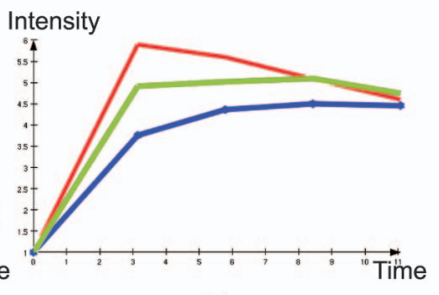

(b)
Fig. 1. (a) TICs for regions of gray matter in the brain (40 measurements). The blue curve shows normal brain perfusion. The red curve indicates no significant perfusion in the infarction core. The green curve shows decreased and delayed perfusion around the core. (b) TICs of different regions in breast tissue (five measurements). The enhancement relative to the signal intensity at the first points in time is shown. The red curve is especially suspicious because of its strong wash-out, which is typical for malignant tumors.

for advanced visualization support. Basic visualization techniques, which are widely available in commercial software, are presented in Section 4. More advanced techniques for the visual exploration, data analysis techniques as well as the combination of both are discussed in Section 5. The application of advanced techniques is described in separate case studies on cerebral perfusion (Section 6), tumor perfusion (Section 7), and myocardial perfusion (Section 8). In Section 9, we propose guidelines for the use of the basic and advanced visualization techniques.

\section{Medical Background}

In perfusion imaging, a certain amount of CA is injected intravenously and its distribution is measured by a repeated acquisition of subsequent images covering the volume of interest [2]. The CA causes signal changes and works as a tracer of perfusion, which means that the uptake of the tracer is relative to the blood flow. The timedependent behavior of the signal depends on the type of CA applied. The CA circulates through the body in several passages until it gets completely excreted. In perfusion imaging, normally only the first pass, which shows the most significant signal changes, is evaluated. Perfusion imaging differs strongly from static imaging, since greater care must be exercised in injection rate and dose, image timing, and image analysis. The diagnostic quality of perfusion data and the derived perfusion parameters depends on the type of CA, the amount of CA, and the speed of injection. Currently, such imaging techniques are mainly performed in a research context [1].

Depending on the physiological process, either the shortterm blood flow or the long-term ( $>1$ minute) diffusion process of the tracer particles through the membranes of the microvessels are represented in the varying signal of the image voxels. Different imaging sequences are used for perfusion imaging: T1-weighted MRI data are typically used for breast cancer diagnosis, whereas T2-weighted MRI data and CT images are employed for the diagnosis of ischemic stroke. In T1-weighted imaging and CT imaging, a signal enhancement is achieved in areas of CA accumulation. In contrast, T2-weighted imaging leads to a decrease of signal intensity where the CA accumulates. To facilitate a consistent processing of the data from both sequencing modalities, the signal intensities in T2-weighted data sets are often inverted prior to any visualization task. This leads to the more intuitive
TABLE 1

Typical Parameters of Data Sets from MRI Perfusion Imaging

\begin{tabular}{|l|c|c|c|c|c|}
\hline & matrix & $R_{S}$ & $D_{S}$ & \# slices & $R_{T}$ \\
\hline \hline Cerebral & $128^{2}$ & 2 & 7 & $10-15$ & $1-2(40-80)$ \\
\hline Breast tumor & $512^{2}$ & 0.7 & 2 & $60-80$ & $60-90(3-10)$ \\
\hline Myocardial & $128^{2}$ & 1.5 & 18 & $3-4$ & $0.5-1(>40)$ \\
\hline
\end{tabular}

The spatial resolution $R_{S}$ and the slice distance $D_{S}$ are given in millimeters, whereas the temporal resolution $R_{T}$ is measured in seconds (the bracketed values represent the number of measurements).

mapping of CA accumulation to signal enhancement (rather than attenuation). The T2-weighted cerebral perfusion data sets used to generate Fig. 1a and Fig. 4 have been inverted. Another MR imaging technique-which is not yet used in clinical routine-is arterial spin labeling. With this noninvasive approach, a CA does not necessarily need to be injected. The CA can be excited endogenous protons [3].

Perfusion data sets from different application areas considerably differ in spatial and temporal resolutions. Table 1 lists typical parameters for data from MR perfusion imaging. In contrast to cerebral and breast tumor perfusion data, which continuously cover the volume of interest, myocardial perfusion data exhibit large gaps (e.g., slice thickness: 6- and 12-mm gaps).

Perfusion parameters. For the diagnosis, regions of interest in healthy and suspicious tissue are defined, and TICs-averaged over all voxels in a selected region-are analyzed. Typical TICs from cerebral and breast tumor perfusions are presented in Fig. 1. The curves observed in myocardial perfusion diagnosis are similar to those of cerebral perfusion. In both application areas, regions exhibiting no significant or a delayed and diminished enhancement (red and green curves in Fig. 1a) are of interest. However, in breast tumor perfusion, regions showing a high early enhancement followed by rapid wash-out, i.e., a decrease of signal intensity afterward, are especially suspicious (red curve in Fig. 1b). To achieve a more quantitative description of curve shape, perfusion parameters are derived from the TICs. Depending on the application area, different sets of perfusion parameters are relevant. However, some parameters are of general interest for almost all application areas (see Fig. 2). Before we describe these parameters, we introduce three auxiliary variables necessary for a reliable evaluation.

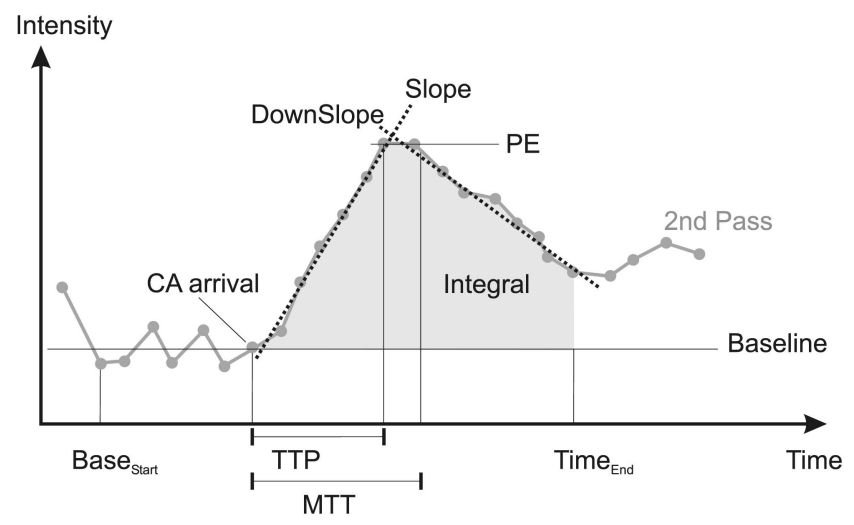

Fig. 2. A typical TIC in myocardial perfusion with a significant first pass and an alleviated second pass of CA traversal annotated with the essential parameters to evaluate the first pass. 
The CA arrival represents the point in time when the signal enhancement actually starts, whereas Time End $_{\text {refers }}$ to the end of the first CA passage. The Baseline represents the average intensity before $C A$ arrival (see Fig. 2). These auxiliary variables are determined to focus the evaluation of the TIC on the relevant portion.

Assessing perfusion considering the actual $C A$ arrival (time), Time End, and the Baseline is essential to compare perfusion analysis results from different scanning devices and patients. Major diagnostically relevant perfusion parameters are given as follows:

- Peak Enhancement (PE). The maximum value normalized by subtracting the baseline.

- Time To Peak (TTP). The point in time where PE occurs, normalized by subtracting the $C A$ arrival time. This parameter allows assessing whether blood supply is delayed in a particular region. If the peak is not a significant maximum or the temporal resolution is low, the TTP value is not expressive. The signal change in the interval between $C A$ arrival and TTP is referred to as wash-in, whereas the signal change in the time between TTP and Time End $_{\text {is }}$ referred to as wash-out.

- Integral. For a certain time interval (often representing one cycle, or pass, of blood flow), the area between the curve and the baseline, which is the approximated integral, is computed. Together, PE and Integral allow one to assess whether the blood supply is reduced in a particular region. Reduced and delayed blood supply is a strong indicator for a damaged region, for example, in ischemic stroke or CHD diagnosis.

- Mean Transit Time (MTT). In the time interval used for the integral calculation, MTT specifies the point in time where the integral is bisected. It is normalized by subtracting $C A$ arrival.

- The Slope characterizes the steepness of the curve during wash-in. Depending on the temporal resolution, different regression methods, such as the Gamma-Variate and a linear fit, are used to characterize the curve progression. The term Up-slope in CHD diagnosis relates to the maximum slope between two or three subsequent time steps between CA arrival and TTP.

- The DownSlope characterizes the steepness of the descending curve during wash-out and is computed similar to the Slope.

Application areas. Throughout this paper, we focus on ischemic stroke, breast tumor, and CHD diagnosis. However, perfusion analysis bears a great potential in other diagnostic tasks as well. As an example, it has been shown that renal perfusion [4] and lung perfusion [5] enhance selected diagnostic processes, such as detecting disorders of pulmonary vessels and acute pulmonary embolism.

Commercial perfusion software. The evaluation of perfusion data is supported by a variety of specific tools dedicated to a particular diagnostic question and to a particular modality. Examples are the PERFUSION 3 SOFTWARE, as package for the General Electric, Advantage Windows workstation, the SIEMENS Syngo, Neuro Perfusion software, and Philips CT perfusion software. The basic features, the

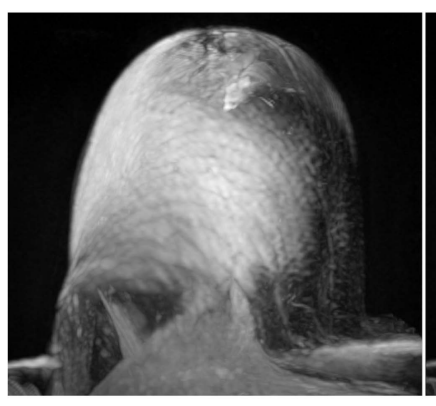

(a)

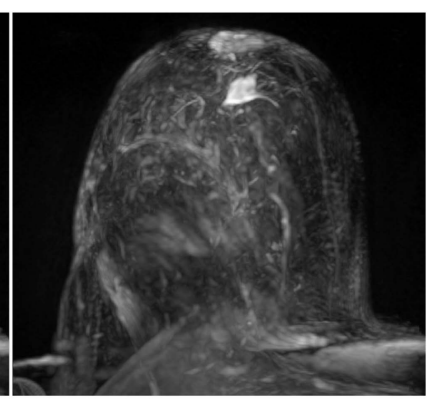

(b)
Fig. 3. Subtraction volumes of dynamic contrast-enhanced MRI mammography data rendered as Maximum Intensity Projection (MIP). (a) Due to respiration, the data exhibit bright artifacts in regions that are not aligned. (b) After aligning the data, the volume becomes more transparent and reveals an enhancing tumor. (Image is courtesy of Sven Kohle, MeVis Research, Bremen. Data are courtesy of Jonathan Wiener, Boca Raton Community Hospital.)

ability to analyze TICs for selected pixels and regions as well as the display of parameter maps are common to all these systems. In nuclear medicine, the EMORY CARDIAC TOOLBOX and Cedars-Sinai's QUANTITATIVE GATEDSPECT SOFTWARE are the two most widely used packages for PET and SPECT data analysis.

\section{Data Processing}

Data processing techniques are experimental, often subject of active research and therefore not widely available. Depending on the specifics of an application area, e.g., the temporal resolution and the amount of motion, data processing techniques have to be applied.

Motion correction. The analysis and visualization of perfusion data relies on comparable image data. Otherwise, subtraction images and TICs are misleading. Comparability means that a voxel with coordinates $(x, y, z)$ at time $t_{1}$ corresponds to a voxel with the same coordinates at time $t_{2}$. Often, a motion correction has to be carried out to achieve comparability. This is essential in assessing tumor and myocardial perfusion where the intervoxel correspondence is hampered due to breathing, patient movement, muscle relaxation, or heartbeat (Fig. 3). Without motion correction, the subtraction volume is filled with bright artifacts. Motion artifacts might hide relevant signal changes but also pretend signal changes that are actually not present.

In dynamic contrast-enhanced (DCE)-MRI mammography, breathing and muscle relaxation result in considerable soft tissue deformations. Here, rigid registration approaches are not appropriate. Elastic registration that considers local transformations enables a better registration quality. The registration algorithm described by Rueckert et al. [6] is a good basis, which employs normalized mutual information [7] as similarity measure. Originally developed for motion correction in DCE-MRI mammography, it is now also used for motion correction in other application areas.

Calibration of signal intensities. While CT imaging provides calibrated signal intensities in "Hounsfield" units, MRI signals are dependent on the scanning sequence used. Therefore, it is very important to calibrate the raw signal 


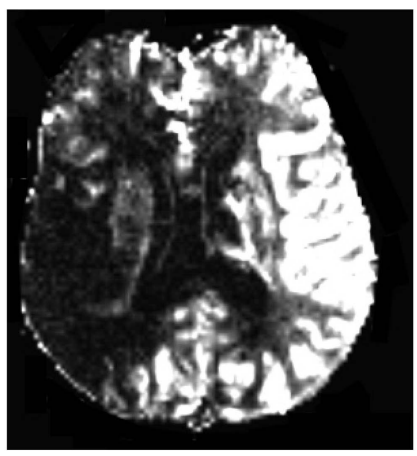

(a)

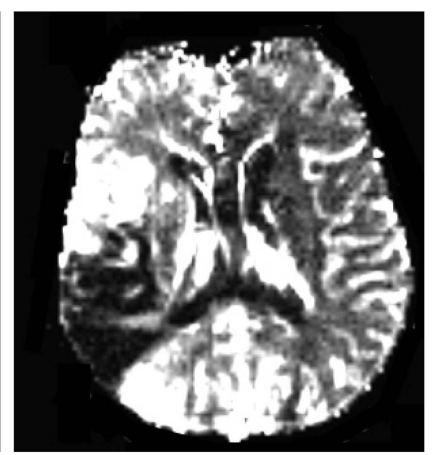

(b)
Fig. 4. Subtraction images to analyze cerebral perfusion. (a) Difference between $t_{6}$ and $t_{2}$. The low perfusion in a larger portion of the right hemisphere (left part in the images) characterizes the infarction zone. (b) Difference between $t_{17}$ and $t_{2}$. The late enhancement in a part of the right hemisphere represents the "tissue at risk." It is characterized by a high signal intensity. (Data are courtesy of Jonathan Wiener, Boca Raton Community Hospital.)

intensities versus CA concentration. For gradient-echo MR sequences, the calibration can be based on the assumption of a linear correspondence between CA concentration and signal intensity [1]. When user-individual vascular input functions are used for the quantitative analysis instead of a standardized assumption about the inflow of CA into the region of interest (ROI), this calibration can be neglected [8]. In this case, input functions correspond to the intensity curves of reference tissue with high vasculature.

Temporal denoising. Since TICs exhibit high-frequency noise, smoothing in the temporal dimension is essential for a reliable analysis. Lysaker et al. [9] introduced an appropriate filter based on partial differential equations, which simulate a diffusion process and applied it to DCEMRI mammography data. For the generation of parameter maps, such as MTT and Integral, the "right" points in time must be chosen (recall Section 2, auxiliary variables). The smoothed visualization supports this selection.

\section{Basic Visualization Techniques}

Some straightforward techniques to visualize and analyze perfusion data are given as follows:

- cine-movies, which step through all points in time for a selected slice,

- subtraction images, depicting the intensity difference between two selected time points,

- color-coded parameter maps for a selected slice. A parameter map depicts the value of a perfusion parameter in a pixelwise manner.

Cine-movies. The cinematic depiction of grayscale images in a movie loop is helpful to assess image noise and artifacts [1] but especially for the assessment of enhancement patterns. In CHD diagnosis, cine-movies are not only applied for assessing myocardial perfusion but also for evaluating the left ventricular wall motion in functional MR data. This special type of data is acquired to image the contraction of the myocardium.

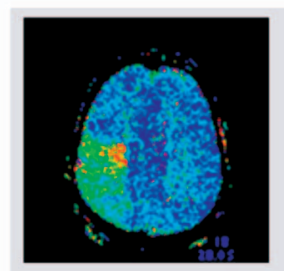

TTP

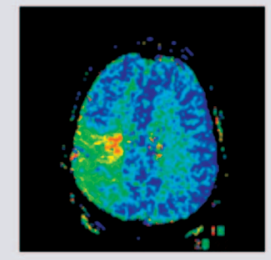

MTT

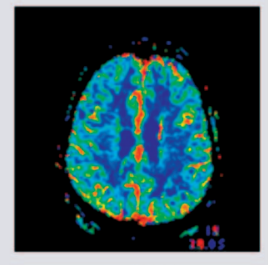

rCBV

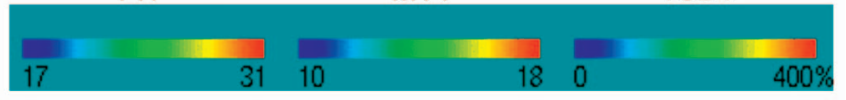

Fig. 5. Parameter maps TTP, MTT, and the relative cerebral blood volume (roughly corresponding to the general perfusion parameter Integral) of a cerebral MRI perfusion data set are depicted. The delayed blood flow in the right hemisphere (left part of the images) becomes obvious. (Images are courtesy of Jonathan Wiener, Boca Raton Community Hospital.)

Subtraction images. Subtraction images may also be used for quality control; the injection of a CA leads to an increase of signal intensity. If the subtraction for two early points in time, $t_{2}$ and $t_{1}$ with $t_{2}>t_{1}$, leads to a negative value, it is likely that the pixels do not correspond to each other due to motion artifacts. If this occurs, motion correction is indispensable for a meaningful analysis. In T2-weighted imaging, where the intensity decreases after $C A$ arrival, the quality control must be adapted (recall Section 2).

In Fig. 4, two subtraction images are shown, which are used for the diagnosis of an ischemic stroke. Both reveal a dark area in the right hemisphere (left part of the images). This is suspicious, since it does not occur in the corresponding region of the left hemisphere. The region, which is dark in both images, depicts the core of an ischemic stroke. Around this region, a larger area appears dark in the early subtraction image (Fig. 4a) but bright in the subtraction image that refers to a later time (Fig. 4b). This region shows the "tissue at risk" around a stroke core.

Subtraction images provide valuable information for the diagnosis. However, there is no assistance in choosing the "right" points in time for subtraction images. Moreover, the 2D data are only used to visually detect abnormalities. Subtraction images do not provide quantitative temporal and spatial information, which could make the diagnostic results more reproducible.

Parameter maps. Parameter maps are displayed as colorcoded images (see Fig. 5). Besides parameter images, it is common to compute TICs for user-selected regions of interest. Often, a parameter map is used first to detect interesting or suspicious regions, whereas TICs in selected regions are analyzed later.

Summary. Basic visualization techniques for exploring perfusion data were described by Behrens et al. [10] and König et al. [11]. Meanwhile, the techniques described above have been integrated into commercial software packages (recall Section 2). The diagnostic quality achieved by using these techniques often depends on appropriate data processing (recall Section 3). 


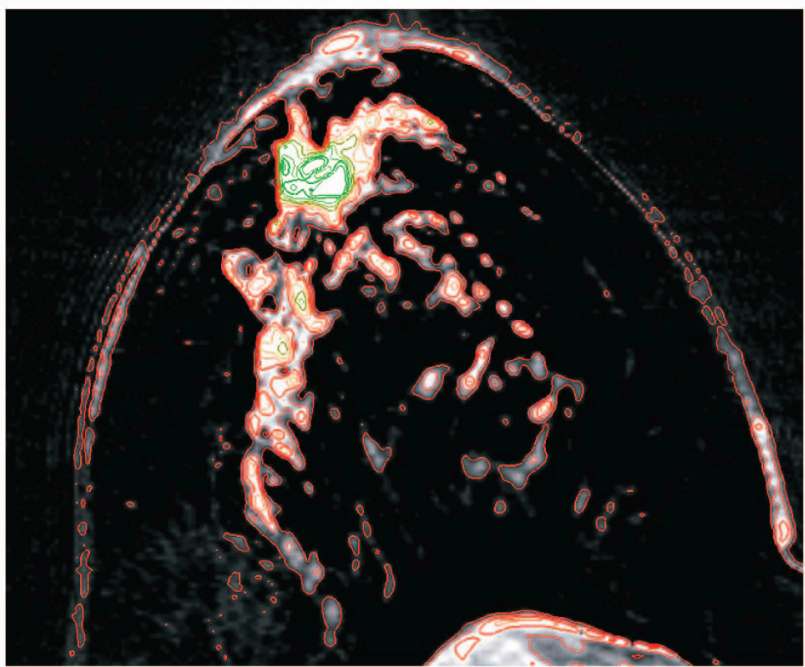

Fig. 6. Isolines corresponding to 10 different isovalues, evenly distributed over the whole range of data, depict a dynamic parameter derived from DCE-MRI mammography. The data and the resulting isolines are smoothed. (Image is courtesy of Olaf Konrad, MeVis Research, Bremen. Data are courtesy of Jonathan Wiener, Boca Raton Community Hospital.)

\section{Advanced Visualization and Analysis TECHNIQUES}

Advanced visualization techniques for exploring perfusion data are motivated by three essential drawbacks of basic visualization techniques. They do not support the integration of

- several parameter maps in one image,

- information derived from perfusion data with morphologic information from another data set,

- extracted features

Sections 5.1, 5.2, and 5.3 show how these problems may be tackled. Data analysis techniques that support the visual exploration by means of classifying tissue according to perfusion characteristics are described in Section 5.4. The combination of data analysis and advanced visualization techniques is presented in Section 5.5.

\subsection{Multiparameter Visualization}

The integrated analysis of several perfusion parameters in a suspicious region is essential for various diagnostic tasks [12]. To support the analysis, we discuss the appropriateness of integrated multiparameter visualizations. These visualizations are based on precomputed parameter volumes, where the corresponding perfusion parameters are represented for each voxel of the original data set.

In principle, color may be employed for two or three parameters as well. Among the widespread color spaces, the HSV space (describing a color by its Hue, Saturation, and Value component) is the best choice since it is perceptually roughly linearized [13]. To be compatible with expectations of users, the most suspicious parameter combinations may be mapped to a red color (Hue) with high saturation and high intensity (Value), whereas normal parameter values are mapped to lower saturation and intensity values and a bluish hue component. With this

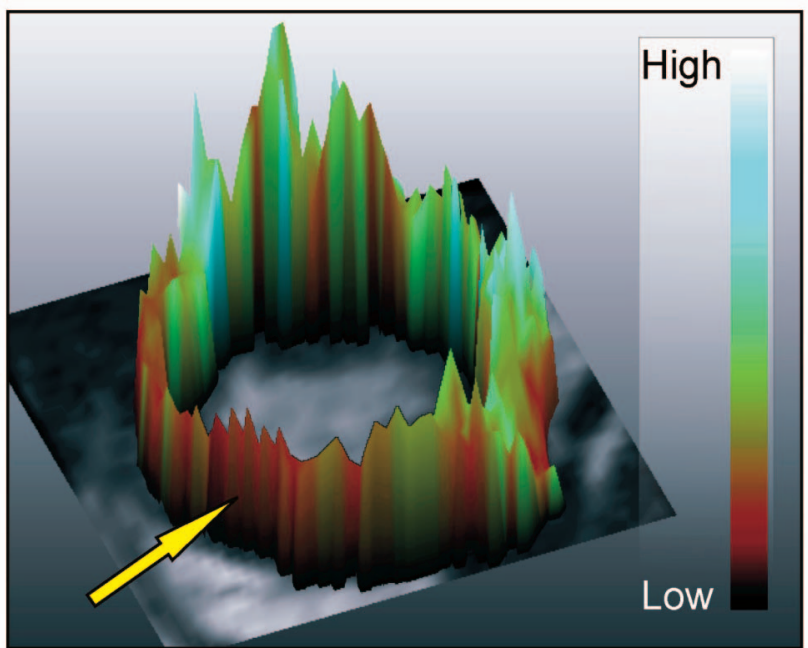

Fig. 7. Colored height field based on parameters $P E$ (height) and Upslope (color). Small elevations (diminished perfusion) and dark colors (delayed perfusion) represent ischemic territories (as pointed at by the arrow) of the segmented myocardium. The corresponding original slice at an adjustable point in time serves as context information. (Data are courtesy of Stefan Miller, University Hospital, Tübingen.)

approach, the viewer's attention is directed to suspicious regions. However, the simultaneous visualization of three quantitative values relating to data with high spatial frequency is in general very hard to interpret. The correct interpretation of two or even three perfusion parameters by means of one color cannot be achieved by preattentive vision. Therefore, Oeltze et al. [14] investigated methods where color (for one perfusion parameter) is combined with another visualization technique for displaying a second parameter. Isolines, height fields, or orientations of textures might be employed to combine several parameters within a single image [15]. In particular, the combination of isolines and colors is effective and can be easily interpreted. Oeltze et al. [14] also discussed the use of color icons [16].

Combining isolines and color coding. Isolines connect regions where the investigated perfusion parameter has a certain value. Isolines are easily computed by the Marching squares algorithm [17]. Noise removal is important in order to prevent that many irrelevant small and distracting isolines or relevant but jaggy lines result (see Fig. 6). In contrast to color coding, isolines are not interpreted at a glance but allow a more quantitative interpretation.

Colored height fields. Colored height fields enable the integrated visualization of two parameter maps. A 3D elevation profile is generated based on the pixel values of the first parameter map. In a next step, the resulting profile is colored according to the pixel values of the second parameter map and an arbitrary color lookup table. The profile may be freely rotated such that initially occluded parts become visible. The mapping to height is scalable. It is initially adapted to the domain of the first parameter (see Fig. 7). As a natural mapping, PE should be mapped to the height parameter.

Exploration of multiple parameter images with lenses. Lenses are used to explore conventional images. Digital lenses-working as pixel magnifiers-are also required in digital image exploration to analyze small-scale phenomena 


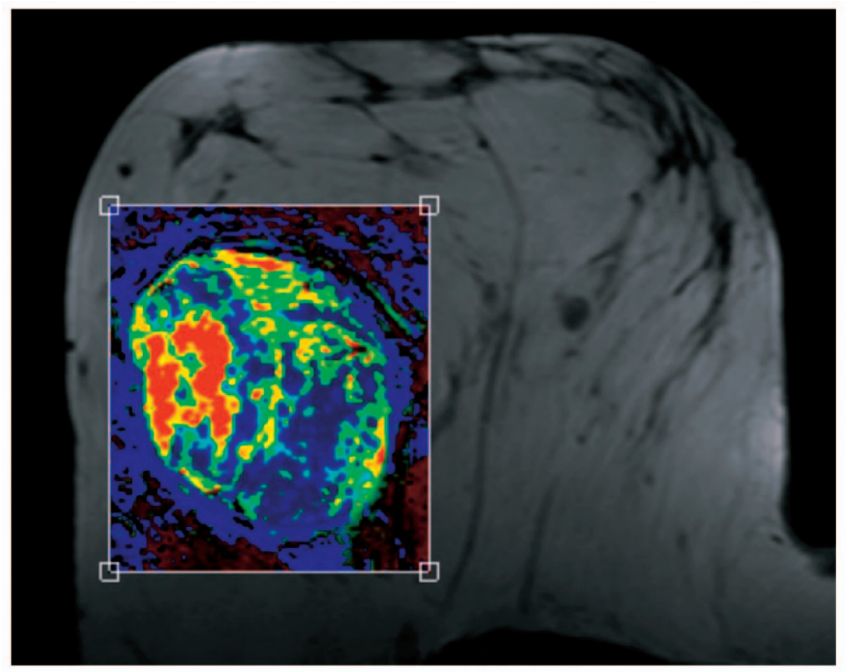

Fig. 8. Exploration of DCE-MRI mammography data with a lens. Parameter DownSlope projected through the lens in the context of the original perfusion data. A blue color indicates a continuous enhancement for a later period in time, a green color indicates a plateau in the TIC. A yellow color and, in particular, a red color indicate a strong washout behavior. (Image is courtesy of Sven Kohle. Data are courtesy of Jonathan Wiener, Boca Raton Community Hospital.)

within enlarged visualizations. The interaction with movable viewing lenses (Magic Lenses) is useful for exploring multidimensional data [18], where lenses do not magnify information but show different information in the lens region. For parameter maps, lenses may show information relating to one parameter either in the context of the original perfusion data (see Fig. 8) or in the context of a map of another parameter (see Fig. 12).

With this interaction style, the user starts by selecting a foreground and a background parameter (for example, TTP and MTT) and then moves a lens (a rectangle or an ellipse) to select either of the parameter set. Inside the lens region, displays of the foreground and background parameter are combined by means of alpha blending (Fig. 12), thus combining an opaque background and translucent foreground to imitate transparency. With an alpha value equal to 1 , only the foreground parameter is represented (Fig. 8).

Glyph-based visualization of multiple parameters. Glyphs represent a standard technique in the visualization of multifield data. A glyph is a simple geometric primitive that is positioned with respect to the original data points in space and whose attributes, e.g., color, extension, size, and orientation, are modified according to some represented values. The integrated glyph-based visualization of multiple perfusion parameters is presented by Oeltze et al. [19] with a focus on an intuitive mapping of perfusion parameter values to glyph shape. Intuitive mapping here refers to the generation of an easy to learn glyph coding of TIC shape, e.g., by mapping parameter Integral (area below the curve) to size and Slope (steepness of the ascending curve) to orientation. Several 2D glyph shapes, e.g., circular discs, ellipses, rectangles, or toroids, with different visual attributes besides color have been implemented in slice-based visualizations. Inspired by the work of Doleisch et al. [20], a feature definition component has been integrated to speed up the evaluation of the complex multifield parameter data.

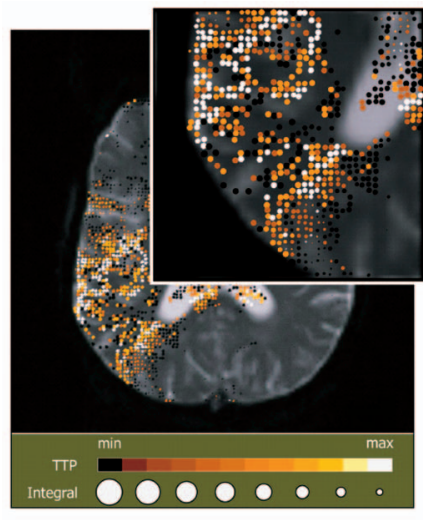

(a)

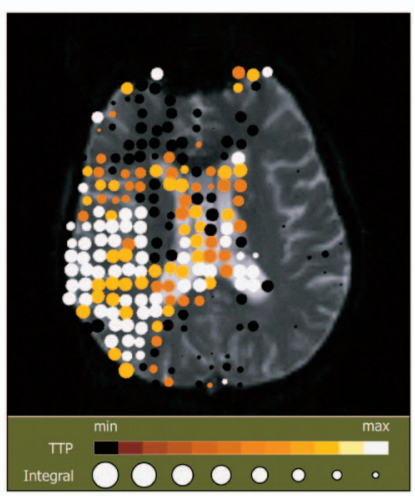

(b)
Fig. 9. Glyph-based visual exploration of cerebral perfusion parameters. The glyph display in all images has been restricted to suspicious regions by means of smooth brushing. (a) One circular disc is placed per data point. Changes in glyph size are hard to interpret. A magnification (inlet) improves the readability but involves a loss of context information and spatial orientation. (b) The application of a lower resolution layer solves the problem. (Data are courtesy of Jonathan Wiener, Boca Raton Community Hospital.)

It is based on the tight integration of the glyph display with multiple statistical representations, connected by (smooth) brushing facilities applied to scatterplot representations. Initial tests showed that the placement of one glyph per data point results in too small glyphs, which is due to the limited screen space (see Fig. 9a). Hence, a multiresolution glyph display has been proposed to improve the readability of the glyph attributes (see Fig. 9b). The display incorporates different resolution layers, which can be interactively explored by the user.

Summary. Multiparameter visualizations enable the simultaneous visualization of two or more perfusion parameters. Height fields are visually attractive and might be used to present diagnostic results, e.g., in educational settings. However, the use of height fields poses interaction problems due to occlusion and is probably not an optimal choice for efficient routine diagnosis. The combination of color and isolines is more promising to support the integrated visualization of two parameters. The use of lenses is based on ideas of clinical users and was considered useful in informal discussions with a neuroradiologist and a cardiologist from the medical faculty of the University of Magdeburg. Both appreciated that they can explore two parameter maps simultaneously instead of having to mentally integrate the information from spatially separated visualizations.

\subsection{Integrating Dynamic Information and Morphology}

Relevant perfusion parameters are often only extracted and visualized for a restricted region in the entire data set, e.g., a tumor or the myocardium. However, other constituents such as the bony structures might provide substantial information for displaying the diagnostically relevant regions in their anatomic environment. Also, other surrounding tissues that are not enhancing can be of indispensable diagnostic value. Therefore, it is useful to add spatial reference information in the regions not containing relevant dynamic information. As 


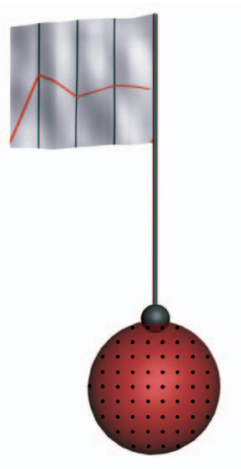

(a)

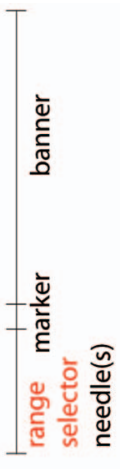

.

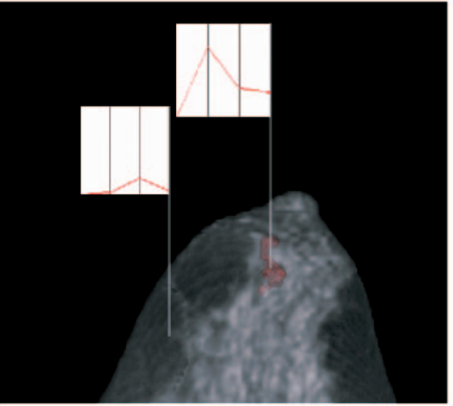

(b)
Fig. 10. (a) Sketch of a Profile Flag. (b) DCE-MRI mammography data with two regions annotated by Profile Flags: (a) a healthy region and (b) a malignant region. The size and shape of the region is determined by the range selector (red polytope). (Data are courtesy of the MR Institut, Univ.-Klinik für Radiodiagnostik Innsbruck, Austria.)

a simple example, the display of an original slice together with the height field in Fig. 7 supports the spatial orientation, which is hampered by the circular shape of the myocardium. A reasonable strategy to add spatial reference information is to color code dynamic information and to display the reference data in the background using a grayscale. Depending on the resolution of the image data, the integration of dynamic and morphologic information should be carried out in $2 \mathrm{D}$ slice visualizations or $3 \mathrm{D}$ renderings. For DCE-MRI mammography data with more than 50 slices, 3D renderings are appropriate, whereas cerebral and myocardial perfusion data provide a too small number of slices.

The assessment of perfusion data might benefit from segmentation information, for example, concerning suspicious breast lesions. In this case, the visualization of dynamic information might be restricted to the segmented region.

\subsection{Probing and Annotating of Perfusion Data}

Another way to depict temporal curves specified at every position of the data is to render them outside of their spatial location. The most common way is to show a set of timedependent graphs for a set of preselected spatial locations, respectively. With this approach, one might loose the correspondence between the spatial position of the measured data and the curve data itself.

Mlejnek et al. [21], [22] proposed the Profile Flag, an intuitive tool for browsing and annotating of temporal data. It enables the visualization of spatial or temporal curves closely connected to the rendering of the anatomic structure of the data without removing any parts thereof. The Profile Flag looks like a board-pin-like glyph, which consists of a banner, a marker, a range selector, and a set of needles (see Fig. 10a). The Profile Flag can be positioned on and dragged along the surface or inside of the inspected anatomical object. For probing of the underlying data, the set of needles is positioned beneath the surface of the probed structure at locations of interest. Each needle defines the position of one probed location or curve. The flagpole is a cylinder that connects the banner with the range selector. The size of the range selector can be changed by moving the marker along the flagpole. The range selector defines the size of the ROI, i.e., it encloses the set of needles. For 3D dynamic perfusion data, the probed information is taken along the time axis (TICs). The temporal development is probed at a specific 3D point location. In the simplest case, this probing position and interesting nearby locations are specified by a spherical range selector. In the more general case, the range selector can be a general polytope including all emphasized TICs. Another way of interaction with the range selectors deals with the selection of TICs based on its properties. One can define more general criteria (e.g., maximal deviation from a predefined reference curve) for the selection of a set of profiles. In this case, the shape of the range selector determines the size and shape of the region, which includes the selected curves (see [22] for details).

The probed curve data is visualized on the banner. Several types of banners can be defined, e.g., a single-profile banner only shows the probed values at the position of a single needle. During the investigation, one or more Profile Flags can be stuck into the inspected object. They can be moved along the object's surface, while showing the underlying probed TICs. Multiple Profile Flags can be placed to emphasize differences between areas from different spatial locations, e.g., healthy versus suspicious regions. For the visualization of dynamic data, the horizontal axis usually corresponds to the time axis. Therefore, for time-varying data, the banner visualizes the time steps along the horizontal axis, while the vertical axis shows the values for each measured time step. Additionally, for sparse temporal data (i.e., just a few time steps), vertical lines are included in order to facilitate reading off the values at particular time steps. Fig. 10b shows an annotation of a DCE-MRI visualization with four time steps by two Profile Flags. The left Profile Flag is located at a healthy region (low increase in the first postcontrast step), while the right Profile Flag illustrates the size and the shape of a suspicious region. The range selector encloses all spatial locations of profiles with similar properties. In this case, the selected set of profiles defines the shape of the range selector. In order to avoid visual clutter, an averaged banner is applied, which shows one curve computed by averaging all probed curves.

The basic concept of Profile Flags has been qualitatively evaluated by the radiologists from Catharina Hospital in Eindhoven [22]. An informative survey indicated that the tool is a valuable add-on to the currently used examination procedures. Furthermore, the complete diagnosis including the anatomy as well as the annotated regions can be printed on one sheet of paper. Thus, one of the essential aspects of the Profile Flags is the efficiency of diagnosis communication between different hospital divisions.

\subsection{Analysis of Perfusion Data}

Another venue of analyzing perfusion data relates to a statistical analysis as well as to mining and knowledge discovery techniques. In particular, the classification of DCE-MRI mammography data by means of artificial neural networks and clustering techniques is an active research area [23], [24], [25]. As an example, Twellmann et al. [25] applied an artificial neural network architecture, which combines unsupervised and supervised techniques for the voxel-byvoxel classification of temporal kinetic signals derived from DCE-MRI mammography data. Chen et al. [23] investigated 


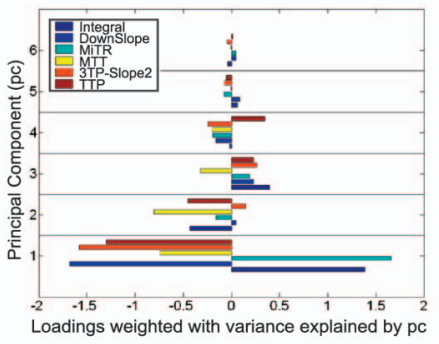

(a)

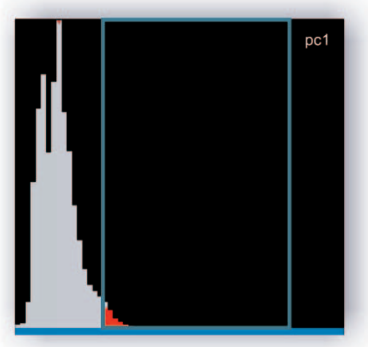

(c)

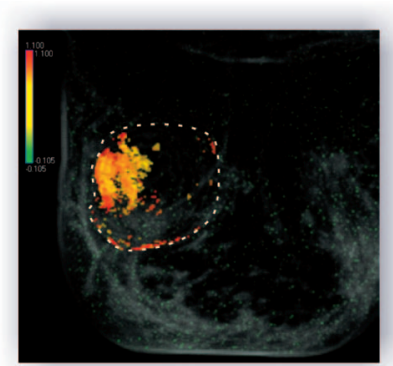

(b)

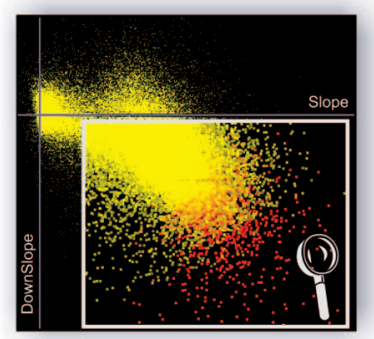

(d)
Fig. 11. Analysis of DCE-MRI mammography data. Examination of the trend represented by the first principal component ( $p c 1$, lower bar chart in (a)). High scores in pc1 have been brushed (b) and the selection is visualized within the context of the right mamma in (c). The selection has been color coded according to Slope. Yellow and red regions indicate as fast wash-in. The boundary of the tumor has been delineated. The selection in (b) is transferred to a scatterplot (d) opposing Slope and DownSlope. Zooming in on the plot reveals that regions exhibiting a fast wash-in as well as a fast wash-out have been detected (red dots). (Data are courtesy of Jonathan Wiener, Boca Raton Community Hospital.)

and developed a fuzzy c-means clustering-based technique to automatically identify characteristic kinetic curves from segmented breast lesions in DCE-MRI mammography data. Nattkemper and Wismüller [26] described the application of self-organized maps to time curve features of DCE-MRI mammography data and discussed how the results may be visually represented as color-coded cross sections. Automatic classification may be useful in a screening setting in order to replace the opinion of a second radiologist or to direct a radiologist to suspicious regions.

\subsection{Combining Analysis and Visual Exploration}

Recently, data analysis techniques and advanced information visualization techniques have been combined in order to efficiently explore the space of perfusion parameters [27]. In particular, a correlation analysis is carried out so as to investigate which perfusion parameters strongly correlate. The remaining parameters are processed by a Principal Component Analysis in order to detect major trends. Inspired by the work of Doleisch et al. [20], the trends as well as the original perfusion parameters are displayed in 2D histograms and scatterplots and are used for (smooth) brushing of relevant subsets of the data (see Fig. 11). This overall strategy turned out to be useful to discriminate different tissues in cerebral perfusion data, tumor perfusion, and myocardial perfusion data.

The overall analysis pipeline is probably too complex for routine diagnosis but may be essential for researchers investigating the effects of CAs, magnetic field strength,

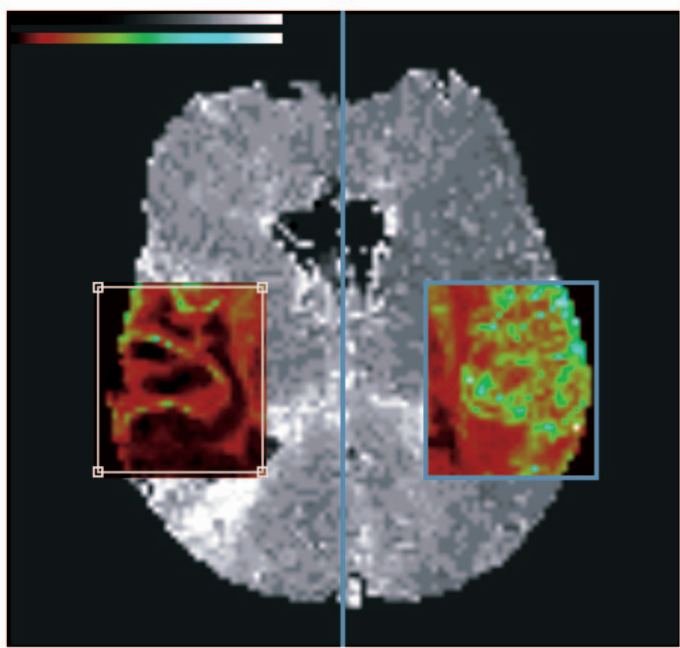

Fig. 12. Synchronized lenses in both hemispheres of the brain support the comparison between the symmetric regions. $P E$ is the foreground parameter mapped to color and TTP is the background parameter. In the lens region, the information from both parameters is integrated by means of alpha blending. The core of the stroke in the right hemisphere (appears left in the image) becomes obvious by comparing the regions inside the synchronized lenses. (Image is courtesy of Christian Bendicks, University of Magdeburg. Data are courtesy of Jonathan Wiener, Boca Raton Community Hospital.)

and other imaging parameters on the diagnostic value of certain perfusion parameter combinations. The pipeline has been discussed with two experienced radiologists from the medical faculty of the University of Magdeburg, both familiar with perfusion imaging in the clinical routine, though not in a research context. Both argued that the data analysis is only applicable in the clinical routine-in particular, in emergency cases-if carried out in the background leading to an initial suggestion for suspicious regions. Both assessed brushing as valuable for exploring a nonstandardized parameter domain. They appreciated the visualization of perfusion data in 3D since it provides a good overview.

The combination of analysis techniques, linking, and brushing for efficiently locating features in perfusion data has been extended with a dense visualization of TICs for all voxels of a perfusion data set. Special techniques are used to reduce clutter in the visualization of a multitude of TICs and dedicated brushes are employed to define TIC target shapes, e.g., a sudden increase and a later decrease of the signal intensity [28].

More specifically, the exploration of higher dimensional histograms for discriminating tissue in ischemic stroke diagnosis was described by Grzesik et al. [29]. They incorporate MR perfusion data and diffusion-weighted MR data in order to integrate the information from both imaging data.

\section{Case Study: Cerebral Perfusion}

In contrast to highly permeable vessels in malignant tumors, microvessels in normal brain tissue do not leak as a result of the blood brain barrier. Consequently, there is no enhancement in the extracellular volume. Instead, we observe the first pass of the CA through the vessel components. About 10 seconds after the first pass of blood circulation, a 
broadened second pass can be seen. The volume of blood in each voxel is diagnostically relevant. It is measured by the Integral parameter of the TIC (see Fig. 2).

CT and MRI are primarily used to assess cerebral perfusion in clinical routine. MRI studies suffer from a lower spatial resolution compared to CT but allow scanning of the entire brain and are thus better suited to detect an infarction, if its location is not a priori known. CT and MRI have both been proven to be useful in diagnosing the acute ischemic stroke and in decision making for therapeutical interventions [30]. However, the feasibility of MRI studies is usually restricted due to the low availability of emergency settings in most clinical institutions, superior costs, and patient-specific difficulties with obtaining MRI, e.g., claustrophobia [30].

Typical parameters for cerebral MRI perfusion data sets are listed in Table 1. In single-slice perfusion CT, one slice covering a brain area of typically $1 \mathrm{~cm}$ is acquired. With multislice CT, an area of $2 \mathrm{~cm}$ may be covered by two or four slices [31]. To reduce image noise, a large slice thickness (one slice with 10-mm thickness or more recently, two slices with 5-mm thickness) is used. The brain coverage may be extended to $4-5 \mathrm{~cm}$ by using two successive bolus administrations [32]. Multislice CT with greater arrays of elements, e.g., 64/256-slice CT, will pave the way for whole brain imaging in clinical routine.

Cerebral perfusion images are used for ischemic stroke diagnosis, in particular to discriminate cerebral hemorrhage and ischemic stroke. In case of an ischemic stroke, the existence and the extent of "tissue at risk" surrounding the core of the stroke has to be evaluated. While the core exhibits no significant perfusion (red curve in Fig. 1a), "tissue at risk" is characterized by a reduced and delayed perfusion (green curve in Fig. 1a). Surgical and chemical interventions may salvage at least parts of the "tissue at risk" [33].

The value of combining cerebral perfusion and diffusion data for predicting stroke evolution is discussed in [34]. In both types of data, areas of ischemic brain tissue can be identified in acute stroke patients. The mismatch between these areas has been reported to present the "tissue at risk" [35]. Sorensen et al. [12] argue that MTT as well as two specific parameters for cerebral perfusion, namely relative cerebral blood volume ( $\mathrm{rCBV}$ ) as well as the relative cerebral blood flow (rCBF), are essential to assess stroke. Wintermark et al. [30] give an overview on seven brain perfusion imaging techniques, including CT and MRI.

Preprocessing cerebral perfusion data is relatively straightforward and primarily involves noise reduction. Breathing and other severe motion artifacts do not occur. The symmetry of the brain is the basis for a diagnostic evaluation of static and dynamic images. Whether or not a part of the brain appears to be pathologic is judged by comparing it with the corresponding part of the other hemisphere. To support symmetry considerations in the exploration of CT and MRI perfusion images, cerebral perfusion tools provide a feature to define an ROI in one hemisphere and let the system define the corresponding ROI in the other hemisphere. The simultaneous display of both TICs (relating to the two regions) supports the evaluation of a correlation between them. In cerebral perfusion diagnosis, synchronized lenses may be used to exploit the symmetry of the brain in axial views. A lens is mirrored on a relocatable, vertical line of symmetry to compare both regions (see Fig. 12 and [36]).

\section{Case Study: Tumor Perfusion}

The process of CA enhancement in a tumor can be described by the diffusion of tracer particles from the inside of blood vessels into the extravascular space and vice versa before it becomes excreted in the kidneys [37]. The permeability of the vessel walls and the extracellular volume fraction determine the amplitude and the shape of the TIC. TICs-which show a high early enhancement followed by a rapid wash-out-are especially suspicious (see red curve in Fig. 1b), because they indicate strong perfusion and high permeability of vessels. Strong perfusion often results from tumor-induced vessel growth (neoangiogenesis). These newly formed vessels are highly permeable, leading to a rapid wash-out [38]. Less suspicious are curves showing a plateau later on (green curve) or regions that continue to enhance (blue curve). This is typically observed in benign tumors.

DCE-MRI mammography has been introduced by Kaiser and Zeitler [39] in 1989. However, only recently the imaging modality gained widespread acceptance, which is partially due to the effective computer support [40]. The major diagnostic task is to confirm or reject the hypothesis of a tumor being malignant. Data processing, in particular motion correction, is challenging (recall Section 3).

In DCE-MRI mammography, T1-weighted images are employed. Contrast enhancement lasts considerably longer than in cerebral blood vessels. Therefore, longer acquisition times are employed. DCE-MRI mammography data are characterized by a high spatial resolution and a low temporal resolution. Typical parameters for DCE-MRI mammography data are listed in Table 1. For more details on tumor perfusion, see [41], [42], and a recent multicenter study [43].

Substantial research to support the diagnosis of DCE-MRI mammography data has been carried out at MeVis Research, Bremen. In the work by Behrens et al. [10], the display of parameter maps, the selection of ROIs, the calculation of TICs, and the quantitative analysis of these curves were presented. Meyer et al. [44] presented a software assistant adapted to the needs of the clinical routine, in particular with respect to the support of breast cancer diagnosis. This assistant has been successfully applied in a clinical trial evaluating the DCEMRI data sets of 46 patients suspected of having breast cancer [45]. With more advanced visualization options, a fast motion correction, and the incorporation of parameters from a pharmacokinetic model, a new research prototype, DynaVision, was presented by Alfke et al. [46]. The prototype was tested in examining the perfusion of pancreatic carcinoma xenografts in mice with severe combined immunodeficiency disease. The findings from perfusion analysis were well correlated with those from histopathological analysis and could be achieved within a reasonably fast time (15 minutes).

A long-term effort on visualizing DCE-MRI mammography data has also been accomplished by Hellwig et al. [47]. They investigated virtual reality input and output technology for exploring stereoscopic 3D visualizations of the original data and derived parameter volumes. They conclude that the stereoscopic display facilitates an improved 


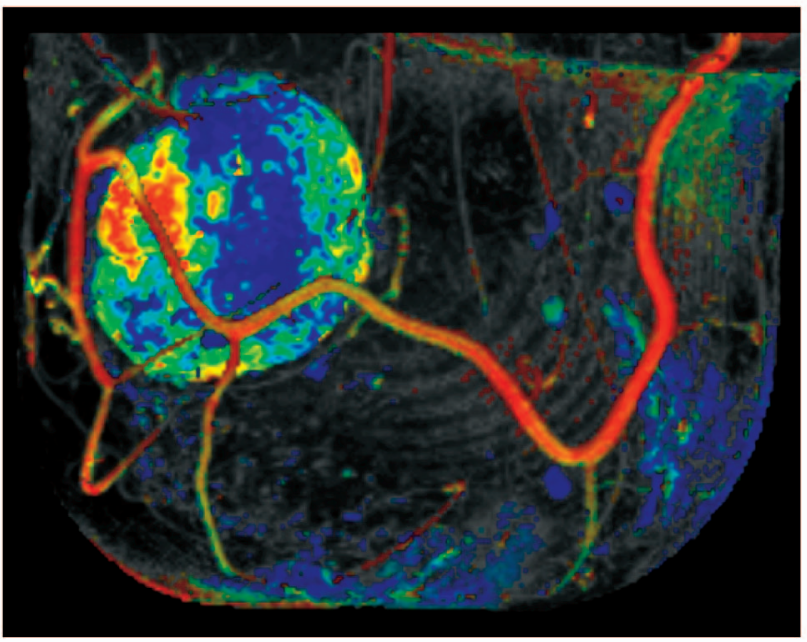

Fig. 13. A grayscale MIP of the subtraction volume of two early points in time is combined with a color-coded CVP. The color encodes the dynamical behavior: bright voxels show a strong enhancement for an early period, less intense voxels show less enhancement. A blue color indicates a continuous enhancement for a later period in time, and a green color indicates a plateau in the TIC. Yellow and red colors indicate a rapid wash-out. (Image is courtesy of Sven Kohle, MeVis Research. Data are courtesy of Jonathan Wiener, Boca Raton Community Hospital.)

localization and differentiation of lesions in space. This is further supported by virtual input devices to adjust, e.g., orientation and transparency of the display. Recently, Coto et al. [48] explored advanced volume rendering techniques for an efficient diagnosis. They have shown that a novel workflow to analyze DCE-MRI mammography data can reduce time needed for diagnosis by utilizing the combination of interactive examination, segmentation, and advanced visualization techniques. Moreover, their software MAMMOEXPLORER allows one to indicate different types of lesions based on both the shape of the lesion and the temporal development of the TIC.

In the following, selected visualization techniques, which have been used for computer support for breast tumor analysis, are described.

Color coding. Two parameters describing the diagnostically significant shape and amplitude of each pixel's TIC may be mapped to color [49]: 1) the slope of the early CA enhancement to brightness and 2) the slope of the late washout to the color value, encoding suspicious wash-out in red. Using continuous color values creates a smooth transition between slowly enhancing and depleting regions.

Projection methods. For the integration of morphologic information and perfusion parameters, 3D visualization techniques are useful. To avoid visual clutter, the visualization of perfusion parameters should be restricted to those voxels exhibiting a high dynamic range (significant signal intensity changes). Projection techniques, such as MIP and Closest Vessel Projection (CVP) [50], provide a direct link between pixels and corresponding voxels with the related TIC. Thus, with a colorized projection image, morphological information can be visualized together with physiological parameters [49] (see Fig. 13).

Kohle et al. [49] suggest to apply a colorized temporal MIP (maximum value along the temporal scale). With this

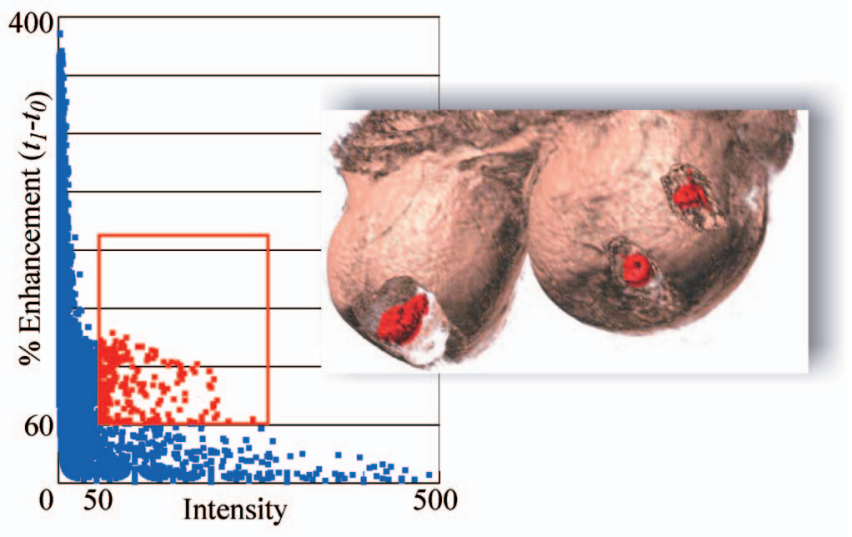

(a)

(b)

Fig. 14. (a) Enhancement scatterplot with a selected region for time step $t_{1}$. (b) Importance-driven volume rendering of areas defined by brushing on a set of enhancement scatterplots. (Images are courtesy of Ernesto Coto, Central University of Venezuela.)

approach, voxels characterized by a strong dynamics (either in the wash-in or wash-out phase) are represented by a color that incorporates the wash-in as well as the wash-out behavior by mapping these values to Hue, Value, and Saturation. As an improvement to MIP, CVP also known as local MIP, was developed to add depth information to MIP images [50]. The most intense voxel along the projection ray is no longer selected; rather, the voxel that represents the first local maximum above a certain threshold is taken. The threshold has to be adjusted to display only the interesting structures. A threshold of 20 percent relative enhancement is appropriate for restricting the visualization to the interesting structures of DCE-MRI mammography data. Fig. 13 shows a colored CVP of DCE-MRI mammography data. Both MIP and CVP are offered as whole-volume visualization techniques and as slab rendering-restricted to a portion of the data characterized by two parallel clipping planes.

Volume rendering and information visualization techniques. The analysis of perfusion data with a large number of slices, such as DCE-MRI mammography data, is a tedious and time-consuming task. It involves browsing through all slices and searching for suspicious areas. Afterward, the procedure is repeated for the identified areas. An effective examination requires the extraction and visualization of diagnostically essential data.

Coto et al. [48] presented several investigation tools (e.g., scatterplots and volume rendering) for the classification and visualization of DCE-MRI mammography data. The approach combines brushing and linking interaction with effective visualization of the selected suspicious areas. For the computation of the TICs, the precontrast scan $\left(t_{0}\right)$ is subtracted from all postcontrast scans $\left(t_{i}\right)$. This step emphasizes the gradient in the temporal dimension of the analyzed curve and highlights suspicious areas. An enhancement scatterplot (see Fig. 14a) is calculated for each postcontrast step. It shows the relative enhancement of the precontrast step with respect to the postcontrast step $t_{i}$. In the interaction step, brushing is performed on one of the scatterplots, while the selected set is emphasized on all remaining scatterplots. If the brushing is performed on 


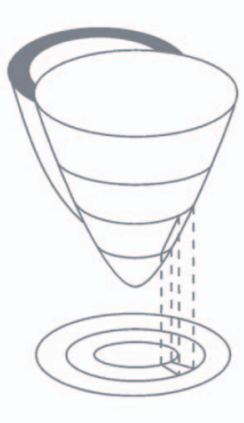

(a)

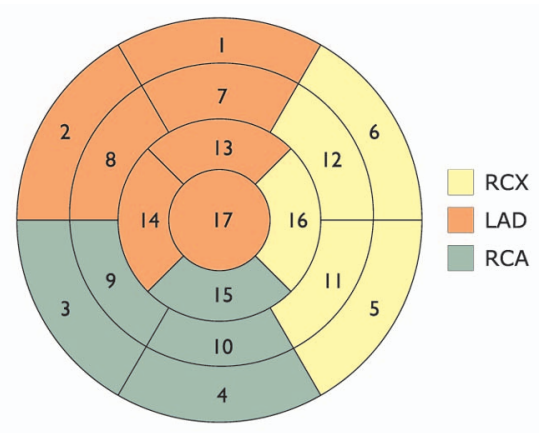

(b)
Fig. 15. (a) AHA conform acquisition of myocardial perfusion data in short-axis views. Schematic representation of the left ventricle that is imaged with three to four slices dissecting the left ventricle basally, centrically, apically, and at the apex. (b) Bull's-Eye Plot and associated AHA conform nomenclature. The plot is generated by projecting the myocardial segments onto a plane. The segments are colored according to the respective supplying coronary branch.

multiple scatterplots, the result of the selection is calculated by a logical "and" operation. The selection set and the way of interaction with the scatterplots depends on the specific application. The result of the interaction can be displayed in a $2 \mathrm{D}$ view by highlighting the selected areas or in a $3 \mathrm{D}$ view with importance-driven volume rendering (see Fig. 14b).

\section{Case Study: Myocardial Perfusion}

Perfusion data is also crucial in the diagnosis of CHD. At an early stage, the CHD is characterized by a perfusion defect caused by a stenosis (an abnormal vessel narrowing). The localization of the perfusion defect with respect to the myocardium combined with anatomical knowledge about the supplying coronary arteries is essential in detecting stenosis as well as in early CHD diagnosis [51]. In particular, for assessing stenosed coronary arteries, imaging modalities from nuclear medicine, such as dynamic PET and SPECT are widespread. With these modalities, the regional distribution of a radioactive isotope, such as $\mathrm{Rb}-82$ chloride, nitrogen 13 (N-13) ammonia, or oxygen 15 (O-15) water, is represented [52], [53], [54], [55].

PET is more specific than SPECT in discriminating vital and irreversibly damaged tissue after an infarction [56]. However, both imaging modalities exhibit a lower spatial resolution and more artifacts, compared to MR and CT perfusion. Also, the amount of radiation for the patient is significantly higher for PET and SPECT data acquisition. Myocardial MR perfusion offers an especially attractive alternative since measures of myocardial perfusion, viability, and ventricular function can be integrated in a single scanning protocol. It has shown to have at least a similar sensitivity and specificity in comparison to PET and SPECT without exposing the patient to radiation [57], [58]. However, widespread use is still hampered by a variety of artifacts and the strong experience necessary to perform the examination and interpret the results. Recent technical advances in hardware, CAs, and imaging sequences are described in [51].

The data acquisition is typically accomplished according to the standards of the American Heart Association (AHA) [59] (Fig. 15a). In MR perfusion imaging, the data acquisition is accomplished during breathhold and it is electrocardiogram (ECG)-triggered over a period of at least 40 consecutive heartbeats. The acquisition is often carried out at rest and under drug-induced stress. The stress test may even reveal marginal stenosis and is usually performed prior to the test at rest using identical imaging parameters. Typical parameters for myocardial perfusion data are listed in Table 1.

According to the AHA standard, the myocardium is divided into 17 segments based on a correspondence between those regions and the supplying coronary branch: ramus circumflex (RCX), left anterior descending (LAD), and right coronary artery (RCA). The perfusion parameters characterizing the CA distribution for each segment are derived from the corresponding averaged TIC. The latter is computed based on the TICs of all voxels in the respective segment. The perfusion parameters are visualized separately by polar coordinates in a color-coded Bull's-Eye Plot (BEP) (Fig. 15b).

For myocardial perfusion diagnosis, the parameters $P E$, TTP, Up-slope, and MTT have been evaluated as especially meaningful [60]. Based on the ratio of the Up-slope at rest and under stress, an additional perfusion parameter, the socalled myocardial perfusion reserve index (MPRI), is computed. The coronary perfusion reserve is defined as the ability of the coronary arteries to increase blood flow under stress by vessel dilation. The MPRI facilitates a more reliable detection of ischemic areas.

The detection and localization of a perfusion deficit as well as the assessment of the severity are directly relevant for treatment decisions. Major diagnostic tasks to be performed are: to evaluate whether the patient suffers from $\mathrm{CHD}$, to evaluate the severity of the disease, and to assess the vascular supply of less perfused tissue. For an overview on MR-based diagnosis of the CHD, see Edelman [61].

The major preprocessing step for myocardial perfusion analysis is motion correction. A combination of rigid and elastic registrations employing mutual information as the similarity measure works reasonably well for most data sets [62]. For parameter computation, the user examines the motion-corrected data set and selects an ROI in healthy myocardial tissue. Utilizing these data, the parameters are calculated for each voxel of the data set. Since the diagnostic questions mostly relate to the myocardium of the left ventricle, the visualization of perfusion parameters is restricted to this structure. The following description is based on the work by Oeltze et al. [14].

Multiparameter visualizations for myocardial perfusion analysis. Multiparameter visualization techniques (Section 5.1) are useful for evaluating myocardial perfusion data (see Fig. 16). The visualization of perfusion parameters by means of colored height fields should be restricted to the segmented myocardium (see Fig. 7).

Refined BEP for rest/stress comparison. In a rest/stress comparison, BEPs (or parameter maps) may be displayed side by side to identify areas where perfusion defects first appear or become worse with stress. In order to simplify a mental integration of rest and stress perfusion in one area, a refined BEP was introduced in [14]. Each segment ring is bisected, thus, duplicating the number of segments. The resulting outer and inner rings represent the stress and the rest states, respectively (Fig. 17). This circular bisection ensures that neighboring segments in the plot are adjacent in the myocardium as well and that they show the same state. Compared to the original variant [14], the segments 


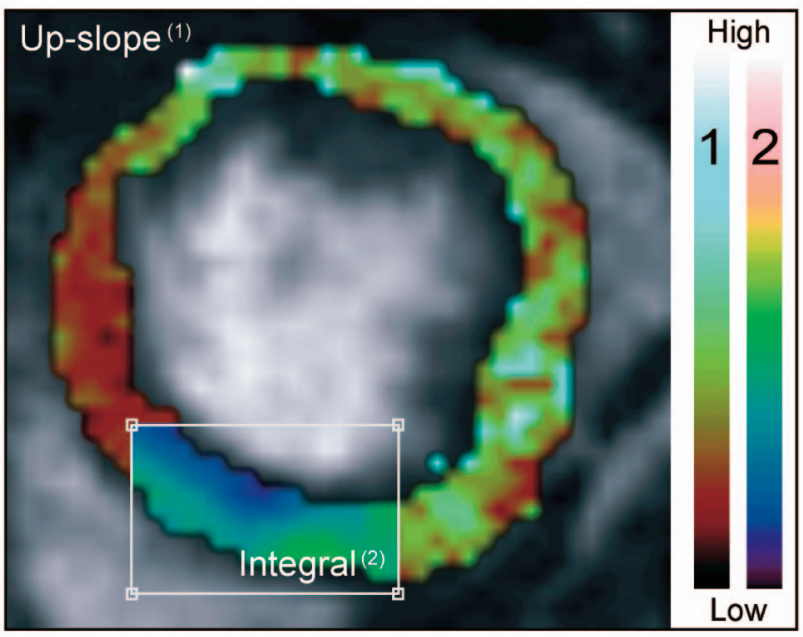

Fig. 16. Parameter Up-slope is displayed for the myocardium in the context of an original slice. Parameter Integral is projected through a user-defined lens. Dark inferior and septal regions indicate a perfusion defect. (Data are courtesy of Stefan Miller, University Hospital, Tübingen.)

are visually separated by a gap (gray ring) for better recognizability. The refined plot may also be used for comparing two different perfusion parameters of one state.

The diagnosis of CHD benefits from a link to morphologic image data, in particular MR/CT angiography data. Oeltze et al. [14] provided a link between the $\mathrm{BEP}$ and the $3 \mathrm{D}$ view of the coronaries and picking facilities for both the plot and the 3D view. A segment of the plot exhibiting a suspicious parameter value may be selected by pointing-resulting in an animated emphasis of the corresponding vessel branch in the 3D view (Fig. 18) and vice versa, emphasizing the supplied segments.

The refined BEP and the linking to morphologic data have been considered useful in informal discussions with a cardiologist from the medical faculty of the University of Magdeburg. He explained that supporting the simultaneous

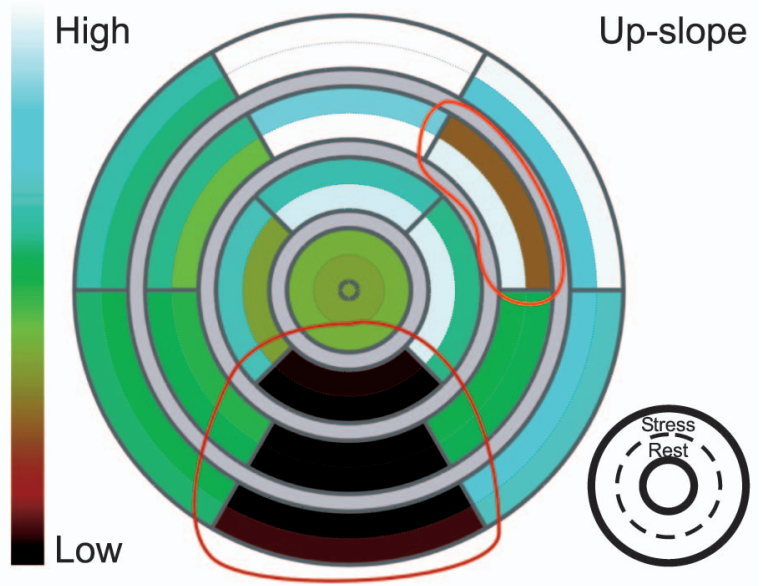

Fig. 17. Integrated visualization of the parameter Up-slope for the rest and stress states in a refined BEP. Dark regions mark the diminished perfusion. An ischemic area is revealed, which spans all inferior segments (lower encircled region). In the anterolateral segment of the mid-cavity (upper encircled region), the perfusion defect may remain unnoticed if perfusion is only examined at rest. (a)

(b)

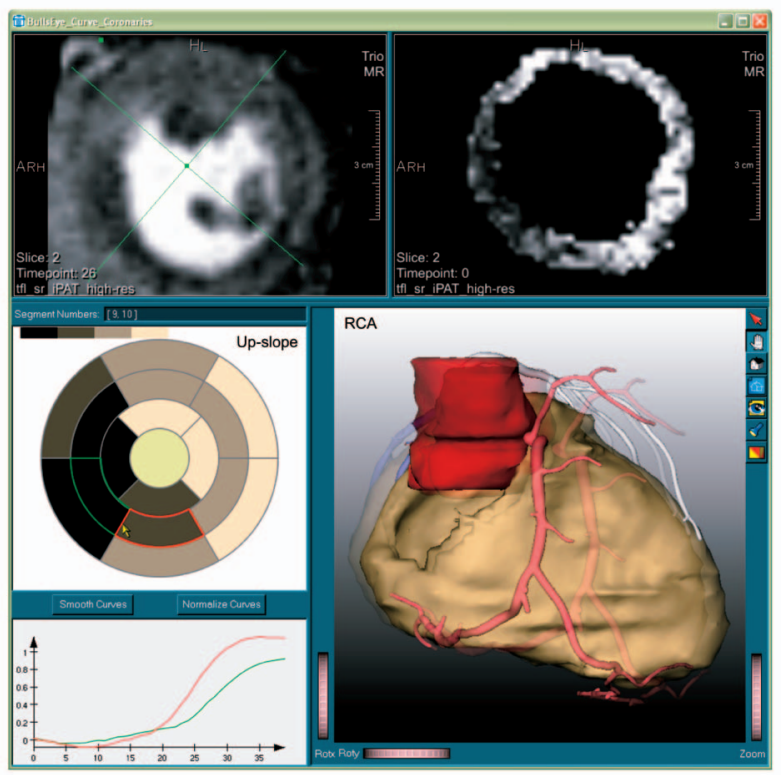

(e)

Fig. 18. Anatomy and myocardial perfusion of a patient suffering from atherosclerosis of the RCA and the LAD. (a) Apical slice of the original perfusion data set and the AHA consistent segmentation of the myocardium overlaid. (b) Apical slice of the parameter volume computed for Up-slope. (c) Selection of two segments in the BEP, which color codes the parameter Up-slope. Segment 17 is missing since no slice has been acquired at the apex itself. (d) TICs corresponding to the selected segments. (e) Coronary branch (RCA) supplying the selected segments. The animated focusing is illustrated by a semitransparent overlay of a previous point in time. (Data are courtesy of Stefan Achenbach, University of Erlangen-Nürnberg.)

examination of rest and stress perfusions has been widely neglected so far. He suggested that the $3 \mathrm{D}$ view would benefit from mapping the perfusion analysis results to the ventricular surface, hereby facilitating an exact assignment of the perfused territories to the supplying coronary branches. However, this requires the registration of the morphologic and the perfusion data.

\section{Guidelines for Visual Exploration}

Over the years, we collected feedback from our clinical collaborators concerning the usefulness of the visual exploration techniques presented in Sections 4 and 5. Based on this feedback, we propose guidelines for the use of these techniques. It turns out that some general guidelines are valid independent of the application area. Before we define the guidelines, we arrange the exploration techniques in three different classes whose separating criterion is the type of required input data:

C1. Input data: the original signal intensities (4D), techniques: cine-movies, subtraction images, and profile flags;

C2. Input data: the perfusion parameters (3D), techniques: parameter maps, multiparameter visualizations, and BEP;

C3. Input data: the original signal intensities and perfusion parameters, technique: MIP+CVP. 
The second class C2 may be further subdivided into two subclasses: C2a (single parameter techniques: parameter maps) and $\mathrm{C} 2 \mathrm{~b}$ (multiparameter techniques: multiparameter visualizations). The original BEP fits into $\mathrm{C} 2 \mathrm{a}$, whereas the refined version belongs to $\mathrm{C} 2 \mathrm{~b}$. The MAMMOEXPLORER (recall Section 7) and the exemplary implementation of the analysis pipeline presented by Oeltze et al. [27] (recall Section 5.5) do not fit in either of these classes. Both rather represent a complex system comprising several techniques and integrate a sophisticated solution to link them.

Techniques of $\mathrm{C} 1$ are useful for an initial inspection of the original data. Tissue characterized by a very strong or by (almost) no enhancement can be roughly identified. Cinemovies require a mental tracking of signal intensity change over time. Their application in breast tumor diagnosis is limited by the low temporal resolution of the data. Profile flags show the signal intensity development plotted in a graph and embedded in a volume rendering, though only for a restricted region. Their application in cerebral and especially in myocardial perfusion is hampered due to the low slice number, preventing a volume rendering. Subtraction images emphasize the highest differences in signal intensity between two time points. However, care must be taken to choose the "right" points. All of the before mentioned techniques require no time-consuming computation or complex user interaction.

After the initial inspection, techniques of C2 may be used for a more thorough analysis of the dynamic behavior in the suspicious tissue. Depending on the number of parameters that are interesting for the clinician, either techniques of class $\mathrm{C} 2 \mathrm{a}$ or $\mathrm{C} 2 \mathrm{~b}$ should be used. The choice of a technique from $\mathrm{C} 2 \mathrm{~b}$ may be influenced by the number of parameters that can be simultaneously displayed, differences in the required computational effort, and the complexity of the required interaction.

Class C3 contains techniques that exploit the original signal intensities as well as the perfusion parameters. The combined analysis of both has already been proven to be useful in a clinical trial for assessing breast tumor perfusion by Wiener et al. [45]. They could show that an analysis based on MIPs of subtraction volumes and parameter maps detected many occult cancers and thereby altered and allowed more confident treatment planning. Kohle et al. [49] proposed an integration of MIP and CVP for the combined analysis. However, this method is not applicable in cerebral and myocardial perfusions due to the low number of slices.

\section{Concluding Remarks}

This paper has discussed general techniques for exploring perfusion data. However, many aspects of the exploration are relevant for other dynamic medical volume data. As an example, the analysis of dynamic Single Photon Emission Computed Tomography (dSPECT) data is also based on the selection of regions and the investigation of curves depicting changes over time in these regions [63]. The analysis of functional MRI data also involves the analysis of time series [64]. The concept of application profile flags, which enable an integrated view of the TICs and the underlying dynamic image data, is also a general approach for exploring a variety of dynamic medical image data [22].
The basic principles of deriving, filtering, and analyzing TICs were developed for the analysis of scintigramms in the 1970s and refined for the analysis of X-ray image sequences [65].

We described a variety of advanced visual analysis techniques that are motivated by discussions with medical doctors and observations of their work that revealed problems with simple visualization techniques. As an obvious drawback, we could not cite any substantial user study related to the superiority of the advanced visualization techniques with respect to specific diagnostic questions. To date, no such user studies exist. Therefore, we conclude that a systematic evaluation of techniques and their parameters as well as combinations is the most important task left open for future work. We also reviewed recent work on data analysis techniques, such as cluster analysis, feature detection, and correlation analysis. Significantly more research is necessary to investigate whether these analysis techniques actually lead to a faster and more accurate interpretation of perfusion data.

\section{ACKNOWLEDGMENTS}

The authors would like to thank Christian Bendicks, Anja Kuss, Lydia Paasche, and Arvid Malyszczyk for implementing some of the described techniques in their Master theses. The authors would also like to thank the substantial support of Ernesto Coto (Central University of Venezuela) and their medical collaborators Frank Grothues (University of Magdeburg), Jonathan Wiener (Boca Raton Community Hospital), Stefan Miller (University Hospital, Tübingen), Stefan Achenbach (University of Erlangen-Nürnberg), and the EasyVision Advanced Development group (Philips Medical Systems). The visual analysis of perfusion data has been accomplished with Philipp Muigg, Helmut Doleisch(VRVis, Vienna), and Helwig Doleisch(University of Bergen, Bergen, Norway). This paper is also based on earlier works of Sven Kohle and Sebastian Meyer (MeVis Research).

\section{REFERENCES}

[1] P. Choyke, A. Dwyer, and M. Knopp, "Functional Tumor Imaging with Dynamic Contrast-Enhanced Magnetic Resonance Imaging," Magnetic Resonance in Medicine, vol. 17, pp. 509-520, 2003.

[2] L. Axel, "Cerebral Blood Flow Determination by Rapid-Sequence Computed Tomography: Theoretical Analysis," Radiology, vol. 137, pp. 679-686, 1980.

[3] J. Detre, J. Leigh, D. Williams, and A. Koretsky, "Perfusion Imaging," Magnetic Resonance in Medicine, vol. 23, no. 1, pp. 37-45, 1992.

[4] H. Michaely et al., "Renal Perfusion: Comparison of SaturationRecovery TurboFLASH Measurements at 1.5T with SaturationRecovery TurboFLASH and Time-Resolved Echo-Shared Angiographic Technique (TREAT) at 3.0T," Magnetic Resonance in Medicine, vol. 24, no. 6, pp. 1413-1419, 2006.

[5] K. Nikolaou et al., "Quantification of Pulmonary Blood Flow and Volume in Healthy Volunteers by Dynamic Contrast-Enhanced Magnetic Resonance Imaging Using a Parallel Imaging Technique," Investigative Radiology, vol. 39, no. 9, pp. 537-545, 2004.

[6] D. Rueckert, L. Sonoda, C. Hayes, D. Hill, M. Leach, and D. Hawkes, "Nonrigid Registration Using Free-Form Deformations: Application to Breast MR Images," IEEE Trans. Medical Imaging, vol. 18, no. 8, pp. 712-721, 1999.

[7] W.M. Wells III, P. Viola, H. Atsumi, S. Nakajima, and R. Kikinis, "Multi-Modal Volume Registration by Maximization of Mutual Information," Medical Image Analysis, vol. 1, no. 1, pp. 35-51, 1996. 
[8] S. Walker-Samuel, M. Leach, and D. Collins, "Reference Tissue Quantification of DCE-MRI Data without a Contrast Agent Calibration," Physics in Medicine and Biology, vol. 52, no. 3, pp. 589-601, 2007.

[9] M. Lysaker, A. Lundervold, and X. Tai, "Noise Removal Using Fourth-Order Partial Differential Equation with Applications to Medical Magnetic Resonance Images in Space and Time," IEEE Trans. Image Processing, vol. 12, no. 12, pp. 1579-1590, 2003.

[10] U. Behrens, J. Teubner, C. Evertsz, M. Walz, H. Jürgens, and H.-O. Peitgen, "Computer-Assisted Dynamic Evaluation of Contrast-Enhanced-MRI," Proc. Computer Assisted Radiology (CAR '96), pp. 362-367, 1996.

[11] M. König, E. Klotz, and L. Heuser, "Perfusion CT in Acute Stroke: Characterization of Cerebral Ischemia Using Parameter Images of Cerebral Blood Flow and Their Therapeutic Relevance," Electromedica, vol. 66, no. 2, pp. 61-67, 1998.

[12] A. Sorensen et al., "Hyperacute Stroke: Simultaneous Measurement of Relative Cerebral Blood Volume, Relative Cerebral Blood Flow, and Mean Tissue Transit Time," Radiology, vol. 210, no. 2, pp. 519-527, 1999.

[13] C. Ware, Information Visualization. Morgan Kaufmann, 2000.

[14] S. Oeltze, F. Grothues, A. Hennemuth, A. Kuß, and B. Preim, "Integrated Visualization of Morphologic and Perfusion Data for the Analysis of Coronary Artery Disease," Proc. Eurographics/IEEE VGTC Symp. Visualization '06, pp. 131-138, 2006.

[15] R. Tyler, "Visualization of Multiple Fields on the Same Surface," IEEE Computer Graphics and Applications, vol. 22, no. 3, pp. 6-10, 2002.

[16] H. Levkowitz, "Color Icons: Merging Color and Texture Perception for Integrated Visualization of Multiple Parameters," Proc. IEEE Visualization (VIS '91), pp. 164-170, 1991.

[17] W. Schroeder, K. Martin, and B. Lorensen, The Visualisation Toolkit, third ed. Kitware, 2001.

[18] E. Bier, M. Stone, K. Pier, W. Buxton, and T. DeRose, "Toolglass and Magic Lenses: The See-Through Interface," Proc. ACM SIGGRAPH '93, pp. 73-80, 1993.

[19] S. Oeltze, A. Malyszczyk, and B. Preim, "Intuitive Mapping of Perfusion Parameters to Glyph Shape," Proc. Bildverarbeitung für die Medizin, pp. 262-266, 2008.

[20] H. Doleisch, M. Gasser, and H. Hauser, "Interactive Feature Specification for Focus + Context Visualization of Complex Simulation Data," Proc. IEEE TCVG/Eurographics Symp. Visualization (EUROVIS '03), pp. 239-248, 2003.

[21] M. Mlejnek, P. Ermes, A. Vilanova, R. van der Rijt, H. van den Bosch, E. Gröller, and F. Gerritsen, "Profile Flags: A Novel Metaphor for Probing of T2 Maps," Proc. IEEE Visualization (VIS '05), pp. 599-606, 2005.

[22] M. Mlejnek, P. Ermes, A. Vilanova, R. van der Rijt, H. van den Bosch, E. Gröller, and F. Gerritsen, "Application-Oriented Extensions of Profile Flags," Proc. Eurographics/IEEE VGTC Symp. Visualization' '06, pp. 339-346, 2006.

[23] W. Chen, M.L. Giger, U. Bick, and G.M. Newstead, "Automatic Identification and Classification of Characteristic Kinetic Curves of Breast Lesions on DCE-MRI," Medical Physics, vol. 33, no. 8, pp. 2878-2887, 2006.

[24] R.E.A. Lucht, M.V. Knopp, and G. Brix, “Classification of SignalTime Curves from Dynamic MR Mammography by Neural Networks," Magnetic Resonance Imaging, vol. 19, no. 1, pp. 51-57, 2001.

[25] T. Twellmann, O. Lichte, and T.W. Nattkemper, "An Adaptive Tissue Characterization Network for Model-Free Visualization of Dynamic Contrast-Enhanced Magnetic Resonance Image Data," IEEE Trans. Medical Imaging, vol. 24, no. 10, pp. 1256-1266, 2005.

[26] T. Nattkemper and A. Wismüller, "Tumor Feature Visualization with Unsupervised Learning," Medical Image Analysis, vol. 9, no. 4, pp. 344-351, 2005.

[27] S. Oeltze, H. Doleisch, H. Hauser, P. Muigg, and B. Preim, "Interactive Visual Analysis of Perfusion Data," IEEE Trans. Visualization and Computer Graphics, vol. 13, no. 6, pp. 1392-1399, Nov./Dec. 2007.

[28] P. Muigg, J. Kehrer, S. Oeltze, H. Piringer, H. Doleisch, B. Preim, and H. Hauser, "A Four-Level Focus + Context Approach to Interactive Visual Analysis of Temporal Features in Large Scientific Data," Computer Graphics Forum, vol. 27, no. 3, pp. 775-782, 2008.
[29] A. Grzesik, J. Bernarding, J. Braun, H.-C. Koennecke, K.J. Wolf, and T. Tolxdorff, "Characterization of Stroke Lesions Using a Histogram-Based Data Analysis Including Diffusion- and Perfusion-Weighted Imaging," Proc. SPIE Medical Imaging: Physiology and Function from Multidimensional Images, vol. 3978, pp. 23-31, 2000.

[30] M. Wintermark et al., "Comparative Overview of Brain Perfusion Imaging Techniques," Stroke, vol. 36, no. 9, pp. 83-99, 2005.

[31] M. Wintermark, N. Fischbein, W. Smith, N. Ko, M. Quist, and W. Dillon, "Accuracy of Dynamic Perfusion CT with Deconvolution in Detecting Acute Hemispheric Stroke," Am. J. Neuroradiology, vol. 26, no. 1, pp. 104-112, 2005.

[32] J. Eastwood, M. Lev, and J. Provenzale, "Perfusion CT with Iodinated Contrast Material," Am. J. Roentgenology, vol. 180, no. 1, pp. 3-12, 2003.

[33] J. den Boer and P. Folkers, "MR Perfusion and Diffusion Imaging in Ischemic Brain Disease," Medica Mundi, vol. 41, no. 2, pp. 20-35, 1997.

[34] S. Rose et al., "MRI Based Diffusion and Perfusion Predictive Model to Estimate Stroke Evolution," Magnetic Resonance Imaging, vol. 19, no. 8, pp. 1043-1053, 2001

[35] S. Warach, J. Gaa, B. Siewert, P. Wielopolski, and R. Edelman, "Acute Human Stroke Studied by Whole Brain Echo Planar Diffusion-Weighted Magnetic Resonance Imaging," Ann. Neurology, vol. 37, no. 2, pp. 231-241, 1995.

[36] S. Oeltze, C. Bendicks, S. Behrens, and B. Preim, "Multiparametervisualisierung zur Exploration Dynamischer Bilddaten," Proc. Bildverarbeitung für die Medizin, pp. 317-321, 2005.

[37] E. Furman-Haran, D. Grobgeld, and H. Degani, "Dynamic Contrast-Enhanced Imaging and Analysis at High Spatial Resolution of MCF7 Human Breast Tumors," J. Magnetic Resonance Imaging, vol. 128, pp. 161-171, 1997.

[38] M. Knopp et al., "Pathophysiologic Basis of Contrast Enhancement in Breast Tumors," Magnetic Resonance in Medicine, vol. 10, pp. 260-266, 1999.

[39] W.A. Kaiser and E. Zeitler, "MR Imaging of the Breast: Fast Imaging Sequences with and without Gd-DTPA-Preliminary Observations," Radiology, vol. 170, pp. 681-686, 1989.

[40] C. Wood, "Computer Aided Detection (CAD) for Breast MRI," Technology in Cancer Research and Treatment, vol. 4, no. 1, pp. 49-53, 2005.

[41] S. Heywang-Köbrunner, P. Viehweg, A. Heinig, and C. Kuchler, "Contrast-Enhanced MRI of the Breast: Accuracy, Value, Controversies, Solutions," European J. Radiology, vol. 24, pp. 94-108, 1997.

[42] H. Degani, V. Gusis, D. Weinstein, S. Fields, and S. Strano, "Mapping Pathophysiological Features of Breast Tumors by MRI at High Spatial Resolution," Nature in Medicine, vol. 2, pp. 780-782, 1997.

[43] M. Schnall et al., "Diagnostic Architectural and Dynamic Features at Breast MR Imaging: Multicenter Study," Radiology, vol. 238, no. 1, pp. 42-53, 2006.

[44] S. Meyer, M. Müller-Schimpfle, H. Jürgens, and H. Peitgen, "MTDYNA: Computer Assistance for the Evaluation of Dynamic MR and CT Data in a Clinical Environment," Proc. Computer Assisted Radiology and Surgery (CARS '99), pp. 331-334, 1999.

[45] J. Wiener, K. Schilling, C. Adami, and N. Obuchowski, "Assessment of Suspected Breast Cancer by MRI: A Prospective Clinical Trial Using a Combined Kinetic and Morphologic Analysis," Am. J. Roentgenology, vol. 184, no. 3, pp. 878-886, 2005.

[46] H. Alfke et al., "Analysis of Mice Tumor Models Using Dynamic MRI Data and a Dedicated Software Platform," Fortschritte auf dem Gebiete der RoÉntgenstrahlen, vol. 176, no. 9, pp. 1226-1231, 2004.

[47] G. Hellwig, K.-H. Englmeier, J. Griebel, R. Lucht, S. Delorme, M. Siebert, and G. Brix, "Dynamic MR Mammography: Multidimensional Visualization of Contrast Enhancement in Virtual Reality," Proc. SPIE Medical Imaging: Physiology and Function from Multidimensional Images, vol. 4683, pp. 54-81, 2002.

[48] E. Coto, S. Grimm, S. Bruckner, E. Gröller, A. Kanitsar, and O. Rodriguez, "MammoExplorer: An Advanced CAD Application for Breast DCE-MRI," Proc. Vision, Modeling, and Visualization (VMV'05), pp. 91-98, 2005.

[49] S. Kohle, B. Preim, J. Wiener, and H.-O. Peitgen, "Exploration of Time-Varying Data for Medical Diagnosis," Proc. Vision, Modeling, and Visualization (VMV'02), pp. 31-38, 2002. 
[50] S. Napel, G. Rubin, and R. Jeffrey, "STS-MIP: A New Reconstruction Technique for CT of the Chest," J. Computer Assisted Tomography, vol. 17, no. 5, pp. 832-838, 1993.

[51] P. Hunold, T. Schlosser, and J. Barkhausen, "Magnetic Resonance Cardiac Perfusion Imaging-A Clinical Perspective," European Radiology, vol. 16, no. 8, pp. 1779-1788, 2006.

[52] H. Iida, I. Kanno, and A. Takahashi, "Measurement of Absolute Myocardial Blood Flow with $\mathrm{H} 215 \mathrm{O}$ and Dynamic Positron Emission Tomography: Strategy for Quantification in Relation to the Partial Volume Effect," Circulation, vol. 78, pp. 104-115, 1988.

[53] A. Kitsiou, S. Bacharach, M. Bartlett, G. Srinivasan, R.M. Summers, A.A. Quyyumi, and V. Dilsizian, "13N-Ammonia Myocardial Blood Flow and Uptake: Relation to Functional Outcome of Asynergic Regions after Revascularization," J. Am. College of Cardiology, vol. 33, no. 3, pp. 678-686, 1999.

[54] S.C. Chua, R.H. Ganatra, D.J. Green, and A.M. Groves, "Nuclear Cardiology: Myocardial Perfusion Imaging with SPECT and PET," Imaging, vol. 18, pp. 166-177, 2006.

[55] M. Merhige, W. Breen, V. Shelton, T. Houston, B. D'Arcy, and A. Perna, "Impact of Myocardial Perfusion Imaging with PET and $(82) \mathrm{Rb}$ on Downstream Invasive Procedure Utilization, Costs, and Outcomes in Coronary Disease Management," European Radiology, vol. 48, no. 7, pp. 1069-1076, 2007.

[56] R. Go, T. Marwick, W. MacIntyre, G.B. Saha, D.R. Neumann, D.A. Underwood, and C.C. Simpfendorfer, "A Prospective Comparison of Rubidium-82 PET and Thallium-201 SPECT in Myocardial Perfusion Imaging Utilizing a Single Dipyridamole Stress in the Diagnosis of Coronary Artery Disease," J. Nuclear Medicine, vol. 31, no. 12, pp. 1899-1905, 1990.

[57] J. Schwitter et al., "Assessment of Myocardial Perfusion in Coronary Artery Disease by Magnetic Resonance: A Comparison with Positron Emission Tomography and Coronary Angiography," Circulation, vol. 103, no. 18, pp. 2230-2235, 2001.

[58] J. Panting, P. Gatehouse, G. Yang, M. Jerosch-Herold, N. Wilke, D. Firmin, and D. Pennell, "Echo-Planar Magnetic Resonance Myocardial Perfusion Imaging: Parametric Map Analysis and Comparison with Thallium SPECT," J. Magnetic Resonance Imaging, vol. 13, no. 2, pp. 192-200, 2001.

[59] M.D. Cerqueira et al., "Standardized Myocardial Segmentation and Nomenclature for Tomographic Imaging of the Heart. A Statement for Healthcare Professionals from the Cardiac Imaging Committee of the Council on Clinical Cardiology of the American Heart Association," Circulation, vol. 105, no. 4, pp. 539-542, 2002.

[60] N. Al-Saadi et al., "Noninvasive Detection of Myocardial Ischemia from Perfusion Reserve Based on Cardiovascular Magnetic Resonance," Circulation, vol. 101, no. 12, pp. 13791383,2000

[61] R. Edelman, "Contrast-Enhanced MR Imaging of the Heart: Overview of the Literature," Radiology, vol. 232, no. 3, pp. 653-668, 2004.

[62] A. Hennemuth, S. Behrens, C. Kuehnel, S. Oeltze, O. Konrad, and H.-O. Peitgen, "Novel Methods for Parameter Based Analysis of Myocardial Tissue in MR-Images," Proc. SPIE Medical Imaging: Physiology, Function, and Structure from Medical Images, vol. 6511, no. $1 \mathrm{~N}$, pp. $1-9,2007$

[63] R. Pohle, M. Wegner, K. Rink, K. Tönnies, A. Celler, and S. Blinder, "Segmentation of the Left Ventricle in 4D-dSPECT Data Using Free Form Deformation of Super Quadrics," Proc. SPIE Medical Imaging: Image Processing, vol. 5370, pp. 1388-1394, 2004.

[64] K. Friston, A. Holmes, J.-B. Poline, P. Grasby, S. Williams, R. Frackowiak, and R. Turner, "Analysis of fMRI Time Series Revisited," NeuroImage, vol. 2, pp. 45-53, 1995.

[65] K. Hoehne, U. Obermoeller, and M. Boehm, "X-Ray Functional Imaging-Evaluation of the Properties of Different Parameters," Proc. Conf. Digital Radiography, pp. 224-228, 1981.

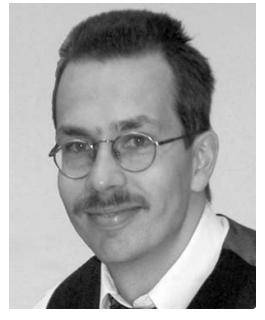

Bernhard Preim received the Diploma in computer science and the $\mathrm{PhD}$ degree in computer science from the University of Magdeburg in 1994 and 1998, respectively, and the Habilitation (venia legendi) from the University of Bremen in 2002. He is currently a professor for visualization in the Department of Simulation and Graphics, Faculty of Computer Science, University of Magdeburg. From 1999 to 2003, he was the head of the Liver Surgery Planning Group, MeVis Research. He is a member of the board of directors of the German Society for Computer- and Roboter-Assisted Surgery (CURAC) and a speaker of the German working group "Visual Computing in Medicine" in the Gesellschaft für Informatik (GI). He is currently a cochair of the Eurographics Workshop on "Visual Computing in Biomedicine." His research interests are in medical visualization (illustrative rendering, visualization of vascular structures, visualization of perfusion data) and applications in diagnosis, surgical education, and surgery planning. $\mathrm{He}$ has authored and coauthored more than 160 conference proceedings and journal papers in these fields and a comprehensive textbook Visualization in Medicine.

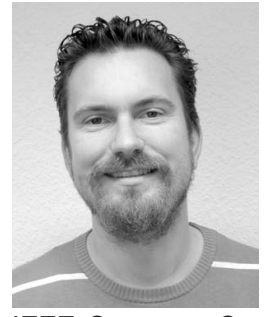

Steffen Oeltze received the Diploma in computational visualistics from the University of Magdeburg in 2004. He is a research and teaching assistant in the Department of Simulation and Graphics, Faculty of Computer Science, University of Magdeburg, Magdeburg, Germany. His research interests are the visual analysis of $4 \mathrm{D}$ perfusion and functional data and the visualization and exploration of vasculature. $\mathrm{He}$ is a student member of the IEEE Computer Society. In 2004/2005, he was distinguished with the "Karl-Heinz-Höhne" Award (second prize) and the 2005 Annual Research Award of the Faculty of Computer Science, University of Magdeburg for his work on vessel visualization. He is a student member of the IEEE Computer Society.

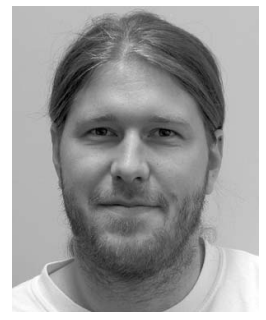

Matej Mlejnek received the $\mathrm{PhD}$ degree in 2006 from Vienna University of Technology, where his thesis is entitled "Medical Visualization for Orthopedic Applications." He is currently a developer at AGFA Healthcare. His main research interests include volume visualization, medical visualization, and image processing.

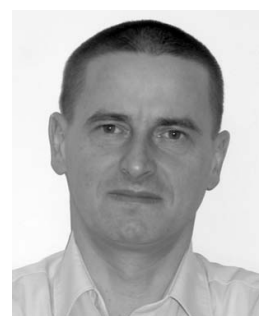

Eduard Gröller is an associate professor at the Institute of Computer Graphics and Algorithms, Vienna University of Technology, Vienna and an adjunct professor of computer science at the University of Bergen, Bergen, Norway. His research interests include computer graphics, flow visualization, volume visualization, medical visualization, and information visualization. $\mathrm{He}$ coauthored more than 150 scientific publications and acted as a cochair, IPC member, and reviewer for numerous conferences and journals in the field. $\mathrm{He}$ is a member of the IEEE Computer Society, the ACM, the Gesellschaft für Informatik (GI), and the Austrian Computer Society (OCG). 


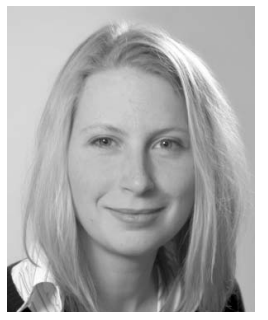

Anja Hennemuth received the MS degree in computer science from the University Hamburg. She is currently a researcher and software developer at MeVis Research, where she develops algorithms and prototypical software applications for the analysis and visualization of cardiac image data in close cooperation with clinical partners from various hospitals.

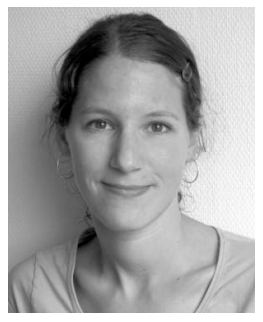

Sarah Behrens received the Diploma in computer science from the University of Bremen in 2003. From 2003 to 2007, she was with MeVis Research, Bremen, as a scientist in medical image processing. Since 2007, she has been a software developer in image-based medicine at MeVis Medical Solutions. Her research interest focused on the analysis of MR images in oncology for diagnosis and therapy planning and breast MR imaging in particular.

$\triangleright$ For more information on this or any other computing topic, please visit our Digital Library at www.computer.org/publications/dlib. 